\title{
Differential Graphical Games for Constrained Autonomous Vehicles Based on Viability Theory
}

\author{
Bowen Peng, Student Member, IEEE, Alexandru Stancu, Member, IEEE, Shuping Dang, Member, IEEE, \\ and Zhengtao Ding, Senior Member, IEEE
}

\begin{abstract}
This paper proposes an optimal distributed control protocol for multi-vehicle systems with an unknown switching communication graph. The optimal distributed control problem is formulated to differential graphical games, and the Pareto optimum to multi-player games is sought based on the viability theory and reinforcement learning techniques. The viability theory characterizes the controllability of a wide range of constrained nonlinear systems; and the viability kernel and the capture basin are the pillars of the viability theory. The capture basin is the set of all initial states, in which there exist control strategies that enable the states to reach the target in finite time, while remaining inside a set before reaching the target. In this regard, the feasible learning region is characterized for the reinforcement learner. In addition, the approximation of the capture basin provides the learner with prior knowledge. Unlike the existing works that employ the viability theory to solve control problems with only one agent and differential games with only two players, the viability theory, in this paper, is utilized to solve multiagent control problems and multi-player differential games. The distributed control law is composed of two parts: the approximation of the capture basin and reinforcement learning, which are computed off-line and on-line respectively. The convergence properties of the parameters' estimation errors in reinforcement learning are proved, and the convergence of the control policy to the Pareto optimum of the differential graphical game is discussed. The guaranteed approximation results of the capture basin are provided, and the simulation results of the differential graphical game are provided for multi-vehicle systems with the proposed distributed control policy.
\end{abstract}

Index Terms-Cooperative control, multi-agent system, capture basin, differential graphical game, reinforcement learning, cooperative learning, viability theory.

\section{INTRODUCTION}

D ISTRIBUTED control techniques and differential graphical games for constrained autonomous robotic systems have drawn considerable attention in the research field of applied engineering for years. The distributed control problem of large complex network systems is always one of the major barriers in industrial applications. Various studies on distributed control have pointed to air traffic control, sensor networks, satellite formation control, multi-robot docking, distributed learning, and so on [1], [2]. The aim of distributed control is to design a control policy for each connected agent such that the evolutions of all the agents will finally contribute to the desired behaviour of the network system

This work is partially supported by the Zhejiang Lab 2019 NB0AB06 and the National Natural Science Foundation of China under Grant 61860206008, 61773081, 61933012, 61833013, 61991403 and 61991400.

B. Peng is with the School of Automation, Chongqing University, Chongqing, 400044, China, and he was with the School of Engineering, Department of Electrical and Electronic Engineering, University of Manchester, Manchester M13 9PL, U.K. (e-mail: bowen.peng@cqu.edu.cn)

A. Stancu and Z. Ding are with the School of Engineering, Department of Electrical and Electronic Engineering, University of Manchester, Manchester M13 9PL, U.K. (e-mail: alexandru.stancu@manchester.ac.uk; zhengtao.ding@manchester.ac.uk).

S. Dang is with Computer, Electrical and Mathematical Science and Engineering Division, King Abdullah University of Science and Technology (KAUST), Thuwal 23955-6900, Kingdom of Saudi Arabia (e-mail: shuping.dang@kaust.edu.sa).
[3]. Representative works for multi-vehicle systems in recent literature can be found in [4]-[7]. Among them, fading, switching and delay phenomenon of wireless channels were concerned in [4]-[6], and optimal distributed control problems were formulated to differential graphical games in [8].

Prior to 1944, in [9], game theory was proposed to build a framework for economic behaviours. Then, the interaction behaviours among players and their corresponding strategies were formulated as a game [10]. The game theory was extended to large-scale network games by introducing graphical models for multi-agent systems, which was referred to as graphical games in [11]. Then, the differential graphical game theory was linked to cooperative control in [8]; this study constructed the representations of local interactions among agents in a large network system based on the graphical game theory. By adopting the principle of dynamic programming, for nonlinear network systems, most papers investigated distributed Nash equilibrium solutions to the differential graphical games [12]-[15]. However, a Nash equilibrium may lead to a worse global performance, as it cannot guarantee the Pareto optimum. To our best knowledge, no comprehensive study has hitherto investigated the Pareto optimum to the differential graphical games for nonlinear systems with bounded control.

Two-player differential games for constrained nonlinear systems have been widely studied through the viability theory, as the viability theory characterizes the numerical solution to the game [16]. Based on the viability theory, qualitative and quantitative problems of both control and differential games 'have been solved [17]. With regard to qualitative problems, the capture basin characterizes a set of initial states for constrained nonlinear systems with proper control such that at least one of their trajectories can hit the target in finite time; the capture basin also characterizes the victory domain of the winner in differential games [17]. With regard to quantitative problems, the approximations of the capture basins and the viability kernels determine the value functions in optimal control of constrained nonlinear systems; they also determine the viscosity solution of the Hamilton-Jacobi-Isaacs equation (HJIE) regarding the differential games [17]. The studies on two-player differential games based on viability theory can be found in the following literature [18]-[23]. Among them, in [18], [19], the intertemporal optimality theorem was associated with the viability theory in terms of differential games, which pioneered the fundamental viability approaches in solving the problems of differential games. Based on [18], [19], the barrier phenomenon of the victory domain was proved in [20], which provided a solution of the HJIE; in [21], the viability properties of differential games was investigated by seeking a viscosity solution of the HJIE; in [22], the optimal time function of the games for constrained nonlinear systems was characterized without any assumptions about controllability. The concepts of the conditional viability kernel and value function for 'qualitative games' were later developed in [23]. In the latest literature, based on the 
viability theory and differential game theory, studies such as [24][27] were limited to two-player differential games modelled by constrained nonlinear differential equations. So far, in the field of the viability theory, no study has yet been carried out that adopts viability approaches to solve differential graphical games.

To fill this gap, we formulate multi-agent control problems to differential graphical games, and propose a novel distributed control policy based on the viability theory. Our algorithm is composed of off-line backward set propagation and on-line cooperative learning. We approximate the capture basin in a guaranteed way by using backward propagation on the target set, which improves the propagation approach proposed in [28], as the value function of the differential games can be approximated during propagation. Moreover, our propagation approach can be applied to the set with arbitrary shape. By combining our new proposed propagation methods with the reinforcement learning technique, our contributions can be mainly categorized into the following aspects: by using just local information of each vehicle, the proposed distributed control strategy converges the Pareto optimum to the differential graphical games for nonlinear system with bounded control despite the unknown switching communication graph; the feasible learning region of states, in which solutions exist, is characterized for the priori knowledge of reinforcement learner; the dimensionality issue is overcome when employing numerical tools through the viability theory; the computation redundancy of the on-line cooperative reinforcement learning is relatively low when compared with the distributed learning algorithms proposed in recent literature, and the computational complexity is not subject to the number of agents in the network system.

The remainder of this paper is organized as follows. Section II outlines the preliminary definitions and notations of the viability theory, differential games and multi-agent systems. Section III formulates the distributed control problem to the differential graphical game associated with the viability theory. Section IV discusses our proposed distributed control policy and provides convergence analysis; Section V provides the results of the approximated capture basin and the simulation results of the game. Section VI concludes this paper and discusses our future work.

\section{Preliminaries}

\section{A. Viability Theory}

Consider a smooth nonlinear dynamic system $F$ with bounded control:

$$
\dot{x}(t)=f(x(t), u(x)), \quad u(x) \in[U],
$$

where $x(t) \in \mathbb{R}^{n}$ is the state vector; $u(x) \in \mathbb{R}^{m}$ is the control input; $[\cdot]$ stands for interval vector $[29] ;[U]=\left[\left[\underline{U}_{1}, \bar{U}_{1}\right], \cdots,\left[\underline{U}_{m}, \bar{U}_{m}\right]\right]^{T}$ is the control constraint. Denote $\varphi\left(\tau, x_{0}, u\right)$ as the state of the system $F$ at time $\tau$ starting from $x_{0}=x(0)$ with control input $u(x)$, and the corresponding trajectory can be written as [30], [31]:

$$
\varphi\left([0, \tau] ; x_{0}, u\right)=\bigcup_{t \in[0, \tau]} \varphi\left(t ; x_{0}, u\right) .
$$

The communication zone is denoted as a closed set $K \subseteq \mathbb{R}^{n}$ and the target is denoted as a closed set $C \subseteq \mathbb{R}^{n}$, where $C \subset K$. The definitions of the viable set and the capture basin are indicated as follows:

Definition 1: As specified in [32], the viable set $K$ is the subset of evolutions that satisfy

$$
K=\{x(t) \mid \forall t \geq 0, x(t) \in K\} .
$$

Definition 2: As specified in [31], [33], the capture basin $\operatorname{Capt}_{F}(K, C)$ is the set of all initial states in $K$ such that at least one of their evolutions reaches the target $C$ in finite time $\tau$ while remaining viable in $K$ for $\forall t \in[0, \tau]$.

\section{B. Differential Games}

Denote $u_{i}$ as the strategy of the $i$ th player and $N$ as the number of players, where $u=\left[u_{1}^{T}, u_{2}^{T}, \cdots, u_{N}^{T}\right]^{T}$. The following definitions distinguish the Nash equilibrium and the Pareto optimum:

Definition 3: As specified in [34], for multi-player games, for $\forall i \in\{1, \cdots, N\}$, the Nash equilibrium $u^{*}=\left[u_{1}^{* T}, u_{2}^{* T}, \cdots, u_{N}^{* T}\right]^{T}$ always fulfils $J_{\mathcal{N}_{i}}\left(x(t), u^{*}\right) \leq J_{\mathcal{N}_{i}}\left(x(t), u_{1}^{*}, \cdots, u_{i}, \cdots, u_{N}^{*}\right)$.

In fact, Nash equilibrium may not guarantee a Pareto optimality of the game.

Definition 4: As specified in [35], the strategy $u^{\star}=$ $\left[u_{1}^{\star T}, u_{2}^{\star T}, \cdots, u_{N}^{\star T}\right]^{T}$ is said to be Pareto optimal for control strategies $u$ if the following relation is fulfilled $\forall i \in\{1, \cdots, N\}$, $J_{\mathcal{N}_{i}}\left(x(t), u^{\star}\right) \leq J_{\mathcal{N}_{i}}(x(t), u)$ and $\exists i \in\{1, \cdots, N\}, J_{\mathcal{N}_{i}}\left(x(t), u^{\star}\right)<$ $J_{\mathcal{N}_{i}}(x(t), u)$.

\section{Multi-Agent Systems}

Suppose the total number of nodes is $N$, the communication topology graph is formed as $\mathcal{G}_{N}=\left(\mathcal{V}_{N}, \mathcal{E}_{N}\right)$ where $\mathcal{V}_{N}=$ $\left\{v_{1}, \cdots, v_{N}\right\}$ represents nodes, and $\mathcal{E}_{N} \subseteq \mathcal{V}_{N} \times \mathcal{V}_{N}$ represents edges. Edge set $\left(v_{i}, v_{j}\right) \in \mathcal{E}_{N}$ denotes information sent to the $i$ th node $v_{i}$ from the $j$ th node $v_{j}$, and correspondingly the $i$ th node $v_{i}$ has an in-degree edge; meanwhile, the $j$ th node $v_{j}$ has an outdegree edge. All the in-degree edges of node $v_{i}$ are represented as $v_{\mathcal{N}_{i}}=\left\{v_{j}\right\}: j \in \mathcal{N}_{i}$, where $\mathcal{N}_{i}=\left\{j \mid\left(v_{i}, v_{j}\right) \in \mathcal{E}_{N}\right\}$. In this paper, except for especial denotation, we always denote $(\cdot)_{\mathcal{N}_{i}}=\left\{(\cdot)_{(i, j)}\right\}$ : $j \in \mathcal{N}_{i}$, where $(\cdot)_{(i, j)}$ is shared by the $i$ th and the $j$ th agents. A sequence of edges is said to be a directed path from $v_{i}$ to $v_{j}$, if they are connected in a graph through the nodes from $v_{i}$ to $v_{j}$. An undirected graph is connected if it contains a directed path that connects all nodes. Adjacency matrix $A=\left\{a_{i j}\right\} \in R^{N \times N}$ maps the whole communication network by weighing each edge on their corresponding entry $a_{i j}$ [36]. Normally, if $\left(v_{j}, v_{i}\right) \in \mathcal{E}_{N}$, then we have $a_{i j}>0$, and otherwise $a_{i j}=0$, and all diagonal entries of adjacency matrix are equal to 0 i.e $a_{i i}=0$ [36].

\section{PROBLEM Formulation}

In this paper, each node represents an autonomous vehicle and we number the target vehicle by $i$ and suppose there are $N$ vehicles, denoting $x=\left[x_{1}^{T}, x_{2}^{T}, \cdots, x_{N}^{T}\right]^{T} \in \mathbb{R}^{N n}$ as the global state, $\theta=\left[\theta_{1}^{T}, \theta_{2}^{T}, \cdots, \theta_{N}^{T}\right]^{T} \in \mathbb{R}^{N}$ as the global orientation where $\theta_{i} \in x_{i}$, and $u=\left[u_{1}^{T}, u_{2}^{T}, \cdots, u_{N}^{T}\right]^{T} \in \mathbb{R}^{N m}$ as the global control. The following assumptions always hold throughout this paper:

Assumption 1: The dynamics of all the autonomous vehicles are assumed to be the same.

Assumption 2: The communication topology is considered to be an unknown switching connected graph with $a_{i j}=a_{j i}=1$ if $\left(v_{j}, v_{i}\right) \in \mathcal{E}_{N}$, otherwise $a_{i j}=a_{j i}=0$. Moreover, $\mathcal{A}=\left\{\alpha_{i j}\right\}$ is denoted as the strictly upper triangular form of $A$, and $|\mathcal{A}|=$ $\sum_{i, j=1}^{N} \alpha_{i j}$.

The network dynamic system for multiple vehicles is decomposed into subsystems in terms of relative dynamics. The relative state between $v_{i}$ and $v_{j}$ is denoted as $e_{i j}$ regarding $x_{i}$ as the origin of the local coordinate frame of $v_{i}$. Denoting $\mathcal{R}(\cdot) \in \mathbb{R}^{n \times n}$ as the clockwise rotation matrix and $\theta_{i} \in \mathbb{R}$ as the global orientation of $v_{i}$, 
then $e_{i j}=\mathcal{R}\left(\theta_{i}\right)\left(x_{j}-x_{i}\right) \in \mathbb{R}^{n}$ and $e_{j i}=-\mathcal{R}\left(\theta_{i j}\right) e_{i j}$, where $\theta_{i j} \in e_{i j}$. For any connected vehicles in pairs, we have

$$
\begin{aligned}
& \dot{e}_{i j}=f_{e}\left(e_{i j}\right)+g_{e}\left(e_{i j}\right) u_{i j}, \\
& \dot{e}_{j i}=f_{e}\left(e_{j i}\right)+g_{e}\left(e_{j i}\right) u_{j i}, \\
& e_{i j}, e_{j i} \in K, \quad u_{i j} \in\left[U_{2}\right],
\end{aligned}
$$

where $u_{i j}=\left[u_{i}^{T}, u_{j}^{T}\right]^{T} \in \mathbb{R}^{2 m} ; U_{n}=\mathbf{1}_{n}[U] ; K$ is a closed set representing the communication zone of $v_{i}$ and $v_{j}$. The above dynamic systems (4a) and (4b) are denoted as $F^{+}$and $F^{-}$, respectively. Then, the following assumption holds:

Assumption 3: The functions $f_{e}\left(e_{i j}\right)$ and $g_{e}\left(e_{i j}\right)$ are uniformly continuous and differentiable. Moreover, both of them satisfy

$$
\begin{array}{llll}
\left\|f_{e}\left(e_{i j}(t)\right)\right\| \leq C_{f}\left(1+\left\|e_{i j}(t)\right\|\right), & \exists C_{f} \in \mathbb{R}, & \forall t \in[0, T], \\
\left\|g_{e}\left(e_{i j}(t)\right)\right\| \leq C_{g}\left(1+\left\|e_{i j}(t)\right\|\right), & \exists C_{g} \in \mathbb{R}, & \forall t \in[0, T],
\end{array}
$$

Notably, either $e_{i j}$ or $e_{j i}$ can be transferred into the local coordinate frame of the other. For a pair of nodes $\left(v_{i}, v_{j}\right)$ connecting by bilateral edges, we have

$$
e_{(i, j)}= \begin{cases}e_{i j}, & \text { for } \theta_{i j} \geq 0, \\ -\mathcal{R}\left(\theta_{i j}\right) e_{i j}, & \text { for } \theta_{i j}<0,\end{cases}
$$

such that $e_{(i, j)}=e_{(j, i)}$ always holds for both $e_{i j}$ and $e_{j i}$ regardless of whether the local coordinate frame is either on $v_{i}$ or $v_{j}$. For higher dimension systems, when $\theta_{i}, \theta_{i j} \in \mathbb{R}^{3}$, we transfer either $e_{i j}$ or $e_{j i}$ such that the sign of one of the elements in $\theta_{(i, j)}$ is always identical.

\section{A. Differential Graphical Games}

Let $g_{e}\left(e_{(i, j)}\right)=\left[g_{e}^{\sharp}\left(e_{(i, j)}\right), g_{e}^{b}\left(e_{(i, j)}\right)\right]$ and $g_{e}^{ \pm}\left(e_{(i, j)}\right)=$ $\left[g_{e}^{+}\left(e_{(i, j)}\right), g_{e}^{-}\left(e_{(i, j)}\right)\right]$, where

$$
\begin{aligned}
& g_{e}^{+}\left(e_{(i, j)}\right)= \begin{cases}g_{e}^{\sharp}\left(e_{(i, j)}\right), & \text { for } \theta_{i j} \geq 0 \\
g_{e}^{b}\left(e_{(i, j)}\right), & \text { for } \theta_{i j}<0,\end{cases} \\
& g_{e}^{-}\left(e_{(i, j)}\right)= \begin{cases}g_{e}^{b}\left(e_{(i, j)}\right), & \text { for } \theta_{i j} \geq 0 \\
g_{e}^{\sharp}\left(e_{(i, j)}\right), & \text { for } \theta_{i j}<0,\end{cases}
\end{aligned}
$$

The error systems $F^{+}$and $F^{-}$are unified to the error system $F_{e}$ shown in (8)

$$
\dot{e}_{(i, j)}=f_{e}\left(e_{(i, j)}\right)+g_{e}^{ \pm}\left(e_{(i, j)}\right) u_{i j}, \quad u_{i j} \in\left[U_{2}\right] .
$$

Define a uniformly continuous and differentiable convex function $\psi_{C}\left(e_{(i, j)}\right)$ which configures $C:=\left\{e_{(i, j)} \in K \mid \psi_{C}\left(e_{(i, j)}\right) \leq 0\right\}$ as the target of system $F_{e}$, and the following assumption holds:

Assumption 4: Assume $e_{(i, j)}(T) \in \partial C$ and $T$ is the terminal time which is determined by capture basin approximation, and $C$ is a V-viable set satisfying [30]:

$$
\begin{aligned}
& \forall e_{(i, j)} \in \partial C, \quad \exists u_{i j} \in\left[U_{2}\right], \\
& \nabla \psi_{C}\left(e_{(i, j)}\right)\left(f_{e}\left(e_{(i, j)}\right)+g_{e}^{ \pm}\left(e_{(i, j)}\right) u_{i j}\right)<0 .
\end{aligned}
$$

The corresponding cost function $J_{e}$ is expressed as

$$
\begin{gathered}
J_{e}\left(e_{(i, j)}(\tau), u_{i j}\right)=\psi_{C}\left(e_{(i, j)}(T)\right)+\int_{\tau}^{T} L\left(e_{(i, j)}(t), u_{i j}(t)\right) d t \\
L\left(e_{(i, j)}(t), u_{i j}(t)\right)=Q\left(e_{(i, j)}(t)\right)+u_{i j}^{T}(t) R_{2} u_{i j}(t),
\end{gathered}
$$

where $Q\left(e_{(i, j)}(\tau)\right): \mathbb{R}^{n} \rightarrow \mathbb{R}^{+}$is a positive definite function; $R_{2}=$ $\left[\begin{array}{ll}R & 0 \\ 0 & R\end{array}\right]$ and $R$ is a positive definite matrix. For the relative error system between $v_{i}$ and $v_{j}$, the value function $V_{e}$ is the viscosity solution of

$$
\begin{aligned}
H_{e}\left(e_{(i, j)}, \nabla V_{e}\left(e_{(i, j)}\right)\right) & =0, \\
V_{e}\left(e_{(i, j)}(T)\right) & =\psi_{C}\left(e_{(i, j)}(T)\right),
\end{aligned}
$$

where

$$
\begin{gathered}
H_{e}\left(e_{(i, j)}, p\right)=\inf _{u_{i j} \in\left[U_{2}\right]}\left\{p\left(f_{e}\left(e_{(i, j)}\right)+g_{e}^{ \pm}\left(e_{(i, j)}\right) u_{i j}\right)\right. \\
\left.+L\left(e_{(i, j)}, u_{i j}\right)\right\} .
\end{gathered}
$$

Express the global error system $F_{E}$ as

$$
\begin{aligned}
\dot{e} & =f_{E}(e)+g_{E}(e) u, \quad u \in\left[U_{N}\right], \\
f_{E}(e) & =\left[f_{e}^{T}\left(e_{(1,2)}\right), \cdots, f_{e}^{T}\left(e_{(i, j)}\right), \cdots, f_{e}^{T}\left(e_{(N-1, N)}\right)\right]^{T}, \\
g_{E}(e) & =\operatorname{diag}\left(\left[g_{e}\left(e_{(1,2)}\right), \cdots, g_{e}\left(e_{(i, j)}\right), \cdots, g_{e}\left(e_{(N-1, N)}\right)\right]\right),
\end{aligned}
$$

where the global error $e=\left[e_{(1,2)}{ }^{T}, \cdots, e_{(i, j)}{ }^{T}, \cdots, e_{(N-1, N)}\right]^{T} \in$ $\mathbb{R}^{|\mathcal{A}| n}$ and its element $e_{(i, j)}$ is the relative error of $v_{i}$ and $v_{j}$ in connection, $f_{E}(e) \in \mathbb{R}^{|\mathcal{A}| n}, g_{E}(e) \in \mathbb{R}^{|\mathcal{A}| n \times|\mathcal{A}| m}$. The global cost function is formulated as

$$
J(e(\tau), u)=\sum_{i, j=1}^{N} \alpha_{i j} J_{e}\left(e_{(i, j)}(\tau), u_{i j}\right),
$$

and the value function $V$ is the viscosity solution of

$$
\begin{aligned}
H(e, \nabla V(e)) & =0, \\
V(e(T)) & =\sum_{i, j=1}^{N} \alpha_{i j} \psi_{C}\left(e_{(i, j)}(T)\right) .
\end{aligned}
$$

So far, the distributed control has been formulated as a differential game problem, and the formulation design from (5) to (8) aims to construct $H_{\mathcal{N}_{i}}$, such that the Pareto optimality can be achieved.

Theorem 1: For the game described in (13) and (14), there exists a Pareto solution $u_{\mathfrak{N}_{i}}^{\star}$ associated with the following decoupled Hamiltonian $H_{\mathcal{N}_{i}}$ :

$$
\begin{aligned}
& H_{\mathcal{N}_{i}}\left(e_{\mathcal{N}_{i}}, p_{\mathcal{N}_{i}}\right)=\inf _{u_{i} \in[U]}\left\{\frac{1}{2} p_{\mathcal{N}_{i}} f_{e_{\mathcal{N}}}\left(e_{\mathcal{N}_{i}}\right)+p_{\mathcal{N}_{i}} g_{\mathcal{N}_{i}}^{+}\left(e_{\mathcal{N}_{i}}\right) u_{i}\right. \\
&\left.+\left|\mathcal{N}_{i}\right| u_{i}^{T} R u_{i}+\frac{1}{2} \sum_{j \in \mathcal{N}_{i}} Q\left(e_{(i, j)}\right)\right\},
\end{aligned}
$$

which guarantees the global optimality of the differential graphical game, where

$$
\begin{aligned}
p_{\mathcal{N}_{i}} & =\left[\cdots, p_{(i, j)}, \cdots\right]: j \in \mathcal{N}_{i}, \\
g_{\mathcal{N}_{i}}^{+}\left(e_{\mathcal{N}_{i}}\right) & =\left[\cdots, g_{e}^{+T}\left(e_{(i, j)}\right), \cdots\right]^{T}: j \in \mathcal{N}_{i}, \\
f_{e_{\mathcal{N}}}\left(e_{\mathcal{N}_{i}}\right) & =\left[\cdots, f_{e}^{T}\left(e_{(i, j)}\right), \cdots\right]^{T}: j \in \mathcal{N}_{i} .
\end{aligned}
$$

Proof: The Hamiltonian $H$ in (15a) is formulated as shown in (18) at the top, where $p=\left[p_{(1,2)}, \cdots, p_{(i, j)}, \cdots, p_{(N-1, N)}\right] \in \mathbb{R}^{|\mathcal{A}| n}$ and

$$
\left\{\begin{array}{l}
\mathcal{N}_{i}^{\sharp}=\left\{j \in \mathcal{N}_{i} \mid \theta_{i j} \geq 0\right\}, \\
\mathcal{N}_{i}^{b}=\left\{j \in \mathcal{N}_{i} \mid \theta_{i j}<0\right\} .
\end{array}\right.
$$

The global Hamiltonian $H$ is decomposed to the decoupled local 


$$
\begin{aligned}
& H(e, p)=\inf _{u \in\left[U_{N}\right]}\{ \frac{1}{2} \sum_{i=1}^{N}\left(\sum_{j \in \mathcal{N}_{i}^{\sharp}} p_{(i, j)}\left(g_{e}^{\sharp}\left(e_{(i, j)}\right) u_{i}+g_{e}^{b}\left(e_{(i, j)}\right) u_{j}\right)+\sum_{j \in \mathcal{N}_{i}^{b}} p_{(i, j)}\left(g_{e}^{b}\left(e_{(i, j)}\right) u_{i}+g_{e}^{\sharp}\left(e_{(i, j)}\right) u_{j}\right)\right. \\
&\left.\left.+\sum_{j \in \mathcal{N}_{i}} p_{(i, j)} f_{e}\left(e_{(i, j)}\right)+\sum_{j \in \mathcal{N}_{i}} L\left(e_{(i, j)}, u_{i j}\right)\right)\right\} \\
&= \frac{1}{2} \inf _{u \in\left[U_{N}\right]}\left\{\sum_{i=1}^{N}\left(\sum_{j \in \mathcal{N}_{i}^{\sharp}} p_{(i, j)} g_{e}^{\sharp}\left(e_{(i, j)}\right)+\sum_{j \in \mathcal{N}_{i}^{b}} p_{(i, j)} g_{e}^{b}\left(e_{(i, j)}\right)\right) u_{i}+\sum_{j=1}^{N}\left(\sum_{i \in \mathcal{N}_{j}^{\sharp}} p_{(j, i)} g_{e}^{\sharp}\left(e_{(j, i)}\right)+\sum_{i \in \mathcal{N}_{j}^{b}} p_{(j, i)} g_{e}^{b}\left(e_{(j, i)}\right)\right) u_{i}\right. \\
&\left.+\sum_{i=1}^{N}\left|\mathcal{N}_{i}\right| u_{i}^{T} R u_{i}+\sum_{j=1}^{N} \sum_{i \in \mathcal{N}_{j}} u_{i}^{T} R u_{i}+\sum_{i=1}^{N} \sum_{j \in \mathcal{N}_{i}}\left(p_{(i, j)} f_{e}\left(e_{(i, j)}\right)+Q\left(e_{(i, j)}\right)\right)\right\} \\
&=\sum_{i=1}^{N} \inf _{u_{i} \in[U]}\left\{\left(\sum_{j \in \mathcal{N}_{i}^{\sharp}} p_{(i, j)} g_{e}^{\sharp}\left(e_{(i, j)}\right)+\sum_{j \in \mathcal{N}_{i}^{b}} p_{(i, j)} g_{e}^{b}\left(e_{(i, j)}\right)\right) u_{i}+\left|\mathcal{N}_{i}\right| u_{i}^{T} R u_{i}+\frac{1}{2} \sum_{j \in \mathcal{N}_{i}}\left(p_{(i, j)} f_{e}\left(e_{(i, j)}\right)+Q\left(e_{(i, j)}\right)\right)\right\}, \\
&=\sum_{i=1}^{N} \inf _{u_{i} \in[U]}\left\{\frac{1}{2} p_{\mathcal{N}_{i}} f_{e_{\mathcal{N}}}\left(e_{\mathcal{N}_{i}}\right)+p_{\mathcal{N}_{i}} g_{\mathcal{N}_{i}}^{+}\left(e_{\mathcal{N}_{i}}\right) u_{i}+\left|\mathcal{N}_{i}\right| u_{i}^{T} R u_{i}+\frac{1}{2} \sum_{j \in \mathcal{N}_{i}} Q\left(e_{(i, j)}\right)\right\},
\end{aligned}
$$

Hamiltonian $H_{\mathcal{N}_{i}}$, and $u_{i}$ only appears once in each $H_{\mathcal{N}_{i}}$, hence the solution of $H_{\mathfrak{N}_{i}}$ guarantees the Pareto optimum.

\section{B. Capture Basin}

In the viability theory, the capture basin characterizes the set of states, in which a solution of a control policy exists such that the states can reach the target. In this paper, we determine the terminal time $T$ in Assumption 4 and estimate $V_{e}$ by computing the capture basin. The links between the viability theory and finite horizon optimal control theory are specified in [32]. Based on that, we see the link between the differential game problem and the capture basin as follows:

Theorem 2: Considering the relative error system $F_{e}$ for only two vehicles $v_{i}$ and $v_{j}$, the value function $V_{e}$ is related to the capture basin $\operatorname{Capt}_{\hat{F}_{e}}\left(\varepsilon_{p}\left(\varphi_{L}\left(\tau, e_{(i, j)}\right)\right), \varepsilon_{p}\left(\varphi_{\psi}\left(\tau, e_{(i, j)}\right)\right)\right)$ by the formula

$$
V_{e}\left(e_{(i, j)}(\tau)\right)=\inf _{\left(\tau, e_{(i, j)}, \zeta\right) \in \operatorname{Capt}_{\hat{F}_{e}}\left(\varepsilon_{p}\left(\varphi_{L}\left(\tau, e_{(i, j)}\right)\right), \varepsilon_{p}\left(\varphi_{\psi}\left(\tau, e_{(i, j)}\right)\right)\right.} \zeta,
$$

where $\varepsilon_{p}\left(\varphi_{L}\left(\tau, e_{(i, j)}\right)\right)$ and $\varepsilon_{p}\left(\varphi_{\psi}\left(\tau, e_{(i, j)}\right)\right)$ are epigraphs of $\varphi_{L}\left(\tau, e_{(i, j)}\right)$ and $\varphi_{\psi}\left(\tau, e_{(i, j)}\right)$, respectively. As specified in [32], an epigraph $\varepsilon_{p}(f(x))$ of a function $f(x)$ is expressed as $\varepsilon_{p}(f(x))=$ $\{(x, y) \mid y \geq f(x)\}$. Then, $\varepsilon_{p}\left(\varphi_{L}\left(\tau, e_{(i, j)}\right)\right)$ and $\varepsilon_{p}\left(\varphi_{\psi}\left(\tau, e_{(i, j)}\right)\right)$ are expressed as follows:

$$
\begin{aligned}
\varepsilon_{p}\left(\varphi_{L}\left(\tau, e_{(i, j)}\right)\right)=\{ & \left(\tau, e_{(i, j)}, \zeta\right) \mid \tau \in[0, T], \\
& \left.e_{(i, j)} \in K, \zeta \geq \int_{\tau}^{T} L\left(e_{(i, j)}(t), u_{i j}(t)\right) d t\right\}, \\
\varepsilon_{p}\left(\varphi_{\psi}\left(\tau, e_{(i, j)}\right)\right)=\left\{\left(\tau, e_{(i, j)}, \zeta\right) \mid \tau=T\right. & \left.e_{(i, j)} \in K, \zeta \geq \psi_{C}\left(e_{(i, j)}(T)\right)\right\}
\end{aligned}
$$

and $\hat{F}_{e}$ is an auxiliary system

$$
\left\{\begin{array}{l}
\dot{e}_{(i, j)}=f_{e}\left(e_{(i, j)}\right)+g_{e}^{+}\left(e_{(i, j)}\right) u_{i}+g_{e}^{-}\left(e_{(i, j)}\right) u_{j}, \\
\dot{\zeta}=-L\left(e_{(i, j)}, u_{i j}\right) \\
u_{i j} \in\left[U_{2}\right], \quad e_{(i, j)} \in K, \quad t \in[0, T]
\end{array}\right.
$$

Proof: Given the auxiliary system $\hat{F}_{e},\left(\tau, e_{(i, j)}, \zeta\right) \in$ $\operatorname{Capt}_{\hat{F}_{e}}\left(\varepsilon_{p}\left(\varphi_{L}\left(\tau, e_{(i, j)}\right)\right), \varepsilon_{p}\left(\varphi_{\psi}\left(\tau, e_{(i, j)}\right)\right)\right)$ stands for the set of initial states $\left(\tau, e_{(i, j)}, \zeta\right)$ satisfying

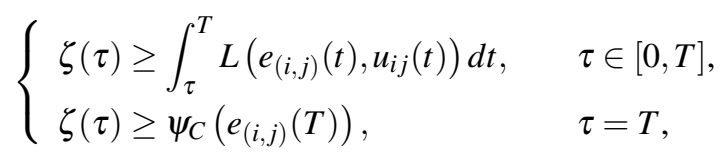

and (23) can be written as, for $\tau \in[0, T]$,

$$
\left\{\begin{array}{l}
\zeta(T) \geq 0, \\
\zeta(\tau) \geq \psi_{C}\left(e_{(i, j)}(T)\right)+\int_{\tau}^{T} L\left(e_{(i, j)}(t), u_{i j}(t)\right) d t,
\end{array}\right.
$$

which implies

$$
\begin{aligned}
V_{e}\left(e_{(i, j)}(\tau)\right) & \leq \psi_{C}\left(e_{(i, j)}(T)\right)+\int_{\tau}^{T} L\left(e_{(i, j)}(t), u_{i j}(t)\right) d t \\
& \left.\leq \inf _{(\tau, e(i, j)}, \zeta\right) \in \operatorname{Capt}_{\hat{F}_{e}}\left(\varepsilon_{p}\left(\varphi_{L}\left(\tau, e_{(i, j)}\right)\right), \varepsilon_{p}\left(\varphi_{\psi}(\tau, e(i, j))\right)\right\} .
\end{aligned}
$$

Given that $V_{e}\left(e_{(i, j)}(T)\right)=\psi_{C}\left(e_{(i, j)}(T)\right) \geq 0$, then

$$
\left\{\begin{aligned}
V_{e}\left(e_{(i, j)}(T)\right)+\varepsilon \geq & 0 \\
V_{e}\left(e_{(i, j)}(\tau)\right)+\varepsilon \geq & \psi_{C}\left(e_{(i, j)}(T)\right) \\
& +\int_{\tau}^{T} L\left(e_{(i, j)}(t), u_{i j}(t)\right) d t
\end{aligned}\right.
$$

and this implies $\left(\tau, e_{(i, j)}, V_{e}\left(e_{(i, j)}(\tau)\right)+\varepsilon\right) \quad \in$ $\operatorname{Capt}_{\hat{F}_{e}}\left(\varepsilon_{p}\left(\varphi_{L}\left(\tau, e_{(i, j)}\right)\right), \varepsilon_{p}\left(\varphi_{\psi}\left(\tau, e_{(i, j)}\right)\right)\right)$ and

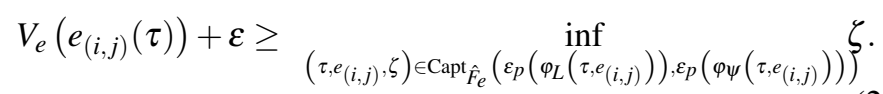

Let $\varepsilon \rightarrow 0$, we summarize from (25) and (27) that

$$
V_{e}\left(e_{(i, j)}(\tau)\right)=\inf _{(\tau, e(i, j), \zeta) \in \operatorname{Capt}_{\hat{F}_{e}}\left(\varepsilon_{p}\left(\varphi_{L}(\tau, e(i, j))\right), \varepsilon_{p}\left(\varphi_{\psi}(\tau, e(i, j))\right)\right.} \zeta .
$$




$$
\begin{aligned}
& \operatorname{Capt}_{\hat{F}_{e}}\left(\varepsilon_{p}\left(\varphi_{L}\right), \varepsilon_{p}\left(\varphi_{\psi}\right)\right)=\{\left(\tau, e_{(i, j)}, \zeta^{\star}\right) \mid \forall \tau \in[0, T], \exists u_{i j}^{\star}(\tau) \in\left[U_{2}\right], e_{(i, j)}(\tau) \in K, \\
&\left.\zeta^{\star}(\tau)=\int_{\tau}^{T} L\left(e_{(i, j)}(t), u_{i j}^{\star}(t)\right) d t, \zeta^{\star}(T)=\psi_{C}\left(e_{(i, j)}(T)\right)\right\} \\
&=\left\{\left(\tau, e_{(i, j)}, \zeta^{\star}\right) \mid \exists \tau \in[0, T], \forall t \in[\tau, T], \exists u_{i j}^{\star}(t) \in\left[U_{2}\right],\right. \\
&\left.\varphi\left([0, T] ; e_{(i, j)}(\tau), u_{i j}^{\star}\right) \subseteq K, \varphi\left(T ; e_{(i, j)}(\tau), u_{i j}^{\star}\right) \in \partial C\right\},
\end{aligned}
$$

Denote $\operatorname{Capt}_{\hat{F}_{e}}^{\star}\left(\varepsilon_{p}\left(\varphi_{L}\right), \varepsilon_{p}\left(\varphi_{\psi}\right)\right)$ as the solution corresponding to $\inf _{i j \in\left[U_{2}\right]} \zeta(\tau): \tau \in[0, T]$, the following theorem holds:

Theorem 3: Under Assumption 3, solving $\operatorname{Capt}_{\hat{F}_{e}}^{\star}\left(\varepsilon_{p}\left(\varphi_{L}\right), \varepsilon_{p}\left(\varphi_{\psi}\right)\right)$ is equivalent to solving $\operatorname{Capt}_{\tilde{F}_{e}}(K, C)$ by Pontryagin Minimum Principle (PMP), where $\tilde{F}_{e}$ stands for the auxiliary system

$$
\begin{gathered}
\left\{\begin{array}{l}
\dot{e}_{(i, j)}=\nabla p_{(i, j)} H_{e}\left(e_{(i, j)}, p_{(i, j)}\right) \\
\dot{p}_{(i, j)}=-\nabla_{e_{(i, j)}} H_{e}\left(e_{(i, j)}, p_{(i, j)}\right) \\
e_{(i, j)}(T) \in \partial C, \quad p_{(i, j)}(T)=\nabla \psi_{C}\left(e_{(i, j)}(T)\right)
\end{array}\right. \\
H_{e}\left(e_{(i, j)}, p_{(i, j)}\right)=\inf _{u_{i j} \in\left[U_{2}\right]}\left\{p_{(i, j)}\left(f_{e}\left(e_{(i, j)}\right)+g_{e}^{ \pm}\left(e_{(i, j)}\right) u_{i j}\right)\right. \\
\left.+L\left(e_{(i, j)}, u_{i j}\right)\right\} .
\end{gathered}
$$

Proof: Given that $C:=\left\{e_{(i, j)} \in K \mid \psi_{C}\left(e_{(i, j)}\right) \leq 0\right\}$ and $e_{(i, j)}(T) \in \partial C, \quad$ for $\forall\left(\tau, e_{(i, j)}, \zeta\right) \in$ $\operatorname{Capt}_{\hat{F}_{e}}\left(\varepsilon_{p}\left(\varphi_{L}\left(\tau, e_{(i, j)}\right)\right), \varepsilon_{p}\left(\varphi_{\psi}\left(\tau, e_{(i, j)}\right)\right)\right)$, we have

$$
\left\{\begin{array}{l}
\inf _{u_{i j} \in\left[U_{2}\right]} \zeta(T)=\psi_{C}\left(e_{(i, j)}(T)\right)=0, \\
\inf _{u_{i j} \in\left[U_{2}\right]} \zeta(\tau)=V_{e}\left(e_{(i, j)}(\tau)\right),
\end{array}\right.
$$

and by PMP, (30) implies that $\forall e_{(i, j)}(T) \in \partial C$ and $\zeta(T)=0$. This means that

$$
\begin{aligned}
\zeta(T) & =\psi_{C}\left(e_{(i, j)}(T)\right) \wedge \zeta(\tau)=\int_{\tau}^{T} L\left(e_{(i, j)}(t), u_{i j}^{\star}(t)\right) d t \\
\zeta(\tau) & =\psi_{C}\left(e_{(i, j)}(T)\right)+\int_{\tau}^{T} L\left(e_{(i, j)}(t), u_{i j}^{\star}(t)\right) d t \\
& =V_{e}\left(e_{(i, j)}(\tau)\right) .
\end{aligned}
$$

Then, let $\zeta^{\star}(\tau)=\inf _{u_{i j} \in\left[U_{2}\right]} \zeta(\tau)$, we have (32) and it can be deduced that $\left(\tau, e_{(i, j)}, \zeta^{\star}\right) \in \operatorname{Capt}_{\hat{F}_{e}}^{\star}\left(\varepsilon_{p}\left(\varphi_{L}\right), \varepsilon_{p}\left(\varphi_{\psi}\right)\right)$, therefore, $e_{(i, j)} \in \operatorname{Capt}_{\tilde{F}_{e}}(K, C)$.

\section{Distributed Control Policy}

Our optimal distributed control law is designed based on the capture basin for the following reasons:

- The capture basin characterizes the set of states, in which solutions exist.

- The capture basin specifies the feasible learning region when adopting reinforcement learning algorithms.

- Approximation of capture basin provides a priori knowledge for the reinforcement learner.

- Solution to the differential game problems is related to the capture basin.

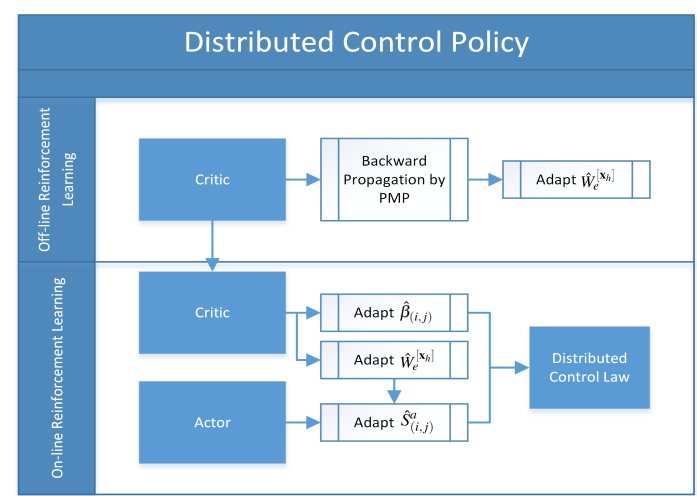

Fig. 1. Flow chart for the design of distributed control policy.

- Reachability of state is guaranteed for the dynamic system with constrained state and bounded control.

The distributed control policy is generated by two stages. The first stage is the approximation of the capture basin $\operatorname{Capt}_{\tilde{F}_{e}}(K, C)$, which initializes the approximation of the value function $V_{e}$ and provides a priori knowledge for the on-line cooperative reinforcement learner. The second stage is the on-line cooperative reinforcement learning, which performs an accurate approximation for the value function $V_{e}$, and optimizes the control policy. The corresponding flow chart is shown in Fig. 1.

\section{A. Backward Propagation}

The capture basin $\operatorname{Capt}_{\tilde{F}_{e}}(K, C)$ is approximated by backward set propagation on $C$. Guaranteed approximations on the set propagation are performed using interval methods. Denote $X$ as the state space of interest; we compose $X$ by smaller non-overlapping interval vectors $\{[\mathbf{x}]\}$ with identical side lengths $d([\mathbf{x}])=\varepsilon_{X}$, where $\varepsilon_{X} \subset \mathbb{R}^{n}$ is a set of resolutions of the state space. Furthermore, every box is numbered with index $h \in \mathbb{N}^{n}$, and sequentially any point $p \in K$ can be localized by the index through a function $\rho(q): \mathbb{R}^{n} \rightarrow \mathbb{Z}^{n}$, where $q \in\left[\mathbf{x}_{h}\right]: h=\rho(q)$. Subpavings are defined as the unions of non-overlapping interval vectors in $X$ [29]. We introduce the following subpavings: $\mathbb{C}, \overline{\mathbb{C}}, \mathbb{K}, \overline{\mathbb{K}}, \operatorname{Capt}_{\tilde{F}_{e}}(K, C)$ and $\overline{\operatorname{Capt}_{\tilde{F}_{e}}(K, C)}$ as follows

Definition 5: $\mathbb{C}, \overline{\mathbb{C}}$ are inner and outer approximations of $C$; $\underline{K}, \overline{\mathbb{K}}$ are inner and outer approximations of $K$; $\operatorname{Capt}_{\tilde{F}_{e}}(K, C)$, $\overline{\operatorname{Capt}_{\tilde{F}_{e}}(K, C)}$ are inner and outer approximations of $\overline{\operatorname{Capt}_{\tilde{F}_{e}}(K, C)}$. 
The relations among them are given by:

$$
\begin{aligned}
& \underline{\mathbb{C}} \subset C \subset \overline{\mathbb{C}} \subset \mathbb{K}, \quad \partial C \subset \partial \mathbb{C}=\overline{\mathbb{C}} \backslash \mathbb{C}, \\
& \underline{\mathbb{K}} \subset K \subset \overline{\mathbb{K}} \subseteq X, \quad \partial K \subset \partial \mathbb{K}=\overline{\mathbb{K}} \backslash \mathbb{K}, \\
& \operatorname{Capt}_{\tilde{F}_{e}}(K, C) \subset \operatorname{Capt}_{\tilde{F}_{e}}(K, C) \subset \overline{\operatorname{Capt}_{\tilde{F}_{e}}(K, C)} \subseteq \underline{\mathbb{K}} .
\end{aligned}
$$

A discrete hypersurface $\mathcal{D}_{0} \in \partial \mathbb{D}_{0}=\partial \mathbb{C}$ is generated to approximate $\partial C$, and all its vertices $\mathbf{Q}_{0}=\left\{q_{0, i}\right\} \subset \partial C$. Its propagated sets are denoted as $\mathcal{D}_{k}$, where $k$ is the retrieve of the iterations. The vertices $\mathbf{Q}_{k}=\left\{q_{k, i}\right\}$ of the $k$ th discrete hypersurface $\mathcal{D}_{k}$ satisfy $q_{k, i} \in\left[\mathbf{x}_{k, i}\right] \backslash \partial\left[\mathbf{x}_{k, i}\right]: i \in\left[1, I_{k}\right]$ and $\partial \mathbb{D}_{k}=\cup\left\{\left[\mathbf{x}_{k, i}\right]\right\}$. Only certain parts of the vertices are chosen to be propagated when they meet the propagation condition. Denote $\mathbb{I}_{k}$ as all of the indexes of the vertices satisfying propagation condition in the $k$ th iteration, $q_{k, i}: i \in \mathbb{I}_{k}$ are chosen to be propagated as their corresponding box $\left[\mathbf{x}_{k, i}\right]: i \in \mathbb{I}_{k}$ satisfies the following condition:

\section{Condition 1:}

$$
\left[f_{e}\right]\left(\left[\mathbf{x}_{k, i}\right]\right)+\left[g_{e}^{ \pm}\right]\left(\left[\mathbf{x}_{k, i}\right]\right) u_{ \pm} \subset \mathcal{T}_{\mathcal{D}_{k}}\left(q_{k, i}\right) \wedge\left[\mathbf{x}_{k, i}\right] \subset \mathbb{K}
$$

where $u_{ \pm}=\left[u_{+}^{T}, u_{-}^{T}\right]^{T},[f](\cdot)$ represents an inclusion function which is expressed in [29]; $\mathcal{T}_{\mathcal{D}_{k}}\left(q_{k, i}\right)$ stands for Bouligand's tangent cone [28], and

$$
u_{ \pm}=\underset{u_{ \pm} \in\left[U_{2}\right]}{\arg \min }\left\{p_{k, i}\left(f_{e}\left(q_{k, i}\right)+g_{e}^{ \pm}\left(q_{k, i}\right) u_{ \pm}\right)+u_{ \pm}^{T} R_{2} u_{ \pm}\right\}
$$

and the control policy $u_{ \pm}$always follows (35) during the propagation. If $\nexists\left[\mathbf{x}_{k, i}\right], i \in\left[1, I_{k}\right]$ satisfies (34), the propagation stops, and the propagation time $t$ determines the terminal time $T$. We denote the retrieve of the terminal iteration as $K_{b}$.

As specified in [32], the backward propagation is denoted as $\overleftarrow{(\cdot)}$, and $\overleftarrow{(\cdot)}(t)=(\cdot)(T-t)$. Given the auxiliary system $\hat{F}_{e}$ and $\tilde{F}_{e}$, by denoting $\phi_{k, i}=\left[\overleftarrow{q}_{k, i}^{T}, \overleftarrow{p}_{k, i}^{T}, \overleftarrow{\zeta}_{k, i}\right]^{T}$, the backward dynamic system $\overleftarrow{F}_{\phi}$ relying on PMP is defined as

$$
\begin{aligned}
\overleftarrow{q}_{k, i}(t)= & -f_{e}\left(\overleftarrow{q}_{k, i}(t)\right)-g_{e}^{ \pm}\left(\overleftarrow{q}_{k, i}(t)\right) u_{ \pm} \\
\overleftarrow{p}_{k, i}(t)= & \overleftarrow{p}_{k, i}(t)\left(\nabla f_{e}\left(\overleftarrow{q}_{k, i}(t)\right)+\nabla g_{e}^{ \pm}\left(\overleftarrow{q}_{k, i}(t)\right) u_{ \pm}\right) \\
& +\nabla_{q_{k, i}} L\left(\overleftarrow{q}_{k, i}(t), u_{ \pm}\right) \\
\overleftarrow{\zeta}_{k, i}(t)= & L\left(\overleftarrow{q}_{k, i}(t), u_{ \pm}\right) \\
\overleftarrow{q}_{0, i}(0) \in & \mathbf{Q}_{0}, \quad \overleftarrow{p}_{0, i}(0)=\nabla \psi_{C}\left(\overleftarrow{q}_{0, i}(0)\right), \quad \overleftarrow{\zeta}_{0, i}(0)=0 .
\end{aligned}
$$

Then, the backward propagation for $\mathcal{D}_{k}$ is performed by

$$
\begin{aligned}
\phi_{k+1, i} & =\varphi_{\overleftarrow{F}_{\phi}}\left(\Delta t_{k} ; \phi_{k, i}, u_{ \pm}\right), \quad i \in \mathbb{I}_{k}, \\
\Delta t_{k} & : \sup _{i \in\left[1, I_{k}\right]}\left\|\varphi_{\overleftarrow{F}_{\phi}}\left(\Delta t_{k} ; \overleftarrow{q}_{k, i}, u_{ \pm}\right)-\overleftarrow{q}_{k, i}\right\|<\min \left\{\varepsilon_{X}\right\}
\end{aligned}
$$

and the propagation time $\Delta t_{k}$ of the $k$ th iteration ensures that the propagated boxes in each loop are connected (i.e. $\partial \mathbb{D}_{k+1} \cap \partial \mathbb{D}_{k} \subset$ $\left.\partial \mathbb{D}_{k}, k \in\left[0, K_{b}-1\right]\right)$, and the proof is similar to [28].

Assume $V^{e}\left(\overleftarrow{q}_{k, i}(t)\right)$ as the true value function for a single relative error system, and $\hat{V}_{e}$ as its approximated value function. As the state space is composed of grids, the approximation is performed piecewise. For $k \in\left[0, K_{b}\right]$ and $i \in\left[1, I_{k}\right]$, assume $\left[\mathbf{x}_{h}\right] \subset \overline{\operatorname{Capt}_{\tilde{F}_{e}}(K, C)} \backslash \underline{\mathbb{C}}$, we have

$$
\begin{aligned}
\hat{V}_{e}\left(\overleftarrow{q}_{k, i}\right) & =\hat{V}_{e}^{\left[\mathbf{x}_{h}\right]}\left(\overleftarrow{q}_{k, i}\right) \\
\hat{V}_{e}^{\left[\mathbf{x}_{h}\right]}\left(\overleftarrow{q}_{k, i}, \hat{W}_{e}^{\left[\mathbf{x}_{h}\right]}\right) & =\hat{W}_{e}^{\left[\mathbf{x}_{h}\right]^{T}} \sigma\left(\overleftarrow{\tilde{q}}_{k, i}\right) \\
V^{e}\left(\overleftarrow{q}_{k, i}\right) & =W_{e}^{\left[\mathbf{x}_{h}\right]^{T}} \sigma\left(\overleftarrow{\tilde{q}}_{k, i}\right)+\varepsilon_{e}^{\left[\mathbf{x}_{h}\right]}\left(\overleftarrow{\tilde{q}}_{k, i}\right)
\end{aligned}
$$

where $\sigma: \mathbb{R}^{n} \rightarrow \mathbb{R}^{l}$ is the activation function of the neural network; $\hat{W}_{e}^{\left[\mathbf{x}_{h}\right]}\left(t_{e}^{\left[\mathbf{x}_{h}\right]}\right) \in \mathbb{R}^{l}$ is the estimated weight of their corresponding activation functions; $t_{e}^{\left[\mathbf{x}_{h}\right]}$ stands for the local time for the parameters assigned to the box $\left[\mathbf{x}_{h}\right]$ and $h=\rho\left(\overleftarrow{q}_{k, i}(t)\right)$; $\overleftarrow{\tilde{q}}_{k, i}(t)=\overleftarrow{q}_{k, i}(t)-\operatorname{mid}\left(\left[\mathbf{x}_{h}\right]\right) ; \operatorname{mid}\left(\left[\mathbf{x}_{h}\right]\right)$ represents the middle point of $\left[\mathbf{x}_{h}\right] ; \varepsilon_{e}^{\left[\mathbf{x}_{h}\right]}$ is the function reconstruction error satisfying $\sup _{\overleftarrow{q}_{k, i} \in\left[\mathbf{x}_{h}\right]}\left\|\varepsilon_{e}^{\left[\mathbf{x}_{h}\right]}\left(\stackrel{(\tilde{q}}{k, i}_{k}\right)\right\| \leq \varsigma_{\varepsilon}^{\left[\mathbf{x}_{h}\right]}$ and $\sup _{\overleftarrow{q}_{k, i} \in\left[\mathbf{x}_{h}\right]}\left\|\nabla \varepsilon_{e}^{\left[\mathbf{x}_{h}\right]}\left(\overleftarrow{\tilde{q}}_{k, i}\right)\right\| \leq \varsigma_{\nabla \varepsilon}^{\left[\mathbf{x}_{h}\right]}$

Our reinforcement learning algorithm is based on actor-critic methods, but only the critic is applied during propagation, where one box corresponds to one critic. Once the first propagated point $\overleftarrow{q}_{k, i}(t)$ enters the box $\left[\mathbf{x}_{h}\right]: h=\rho\left(\overleftarrow{q}_{k, i}(t)\right)$, we initialize the parameters $\Gamma_{e}^{\left[\mathbf{x}_{h}\right]}, \hat{W}_{e}^{\left[\mathbf{x}_{h}\right]}$ and $\omega_{e}(t)$ for the critic of the local box, where $\Gamma_{e}^{\left[\mathbf{x}_{h}\right]} \in \mathbb{R}^{l \times l}$ is initialized to a positive definite matrix, and $\hat{W}_{e}^{\left[\mathbf{x}_{h}\right]}$ and $\omega_{e}(t)$ will be initialized such that

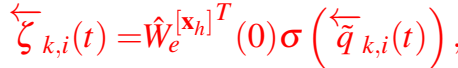

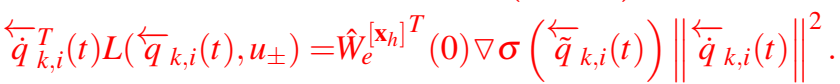

The parameters for a box are all denoted as $(\cdot)_{e}^{\left[\mathbf{x}_{h}\right]}$, and for each box they have their own local time $t_{e}^{\left[\mathbf{x}_{h}\right]}$. When $\overleftarrow{q}_{k, i}(t) \in\left[\mathbf{x}_{h}\right]$, the parameters for the box $\left[\mathbf{x}_{h}\right]$ are updated, and meanwhile the local timer $t_{e}^{\left[\mathbf{x}_{h}\right]}$ is running. Hence, although global time $t$ is always running, if $\overleftarrow{q}_{k, i}(t) \notin\left[\mathbf{x}_{h}\right]$, the local time $t_{e}^{\left[\mathbf{x}_{h}\right]}$ freezes until any point enters the box $\left[\mathbf{x}_{h}\right]$. Let

$$
\begin{aligned}
& \omega_{e}(t)=-\nabla \sigma\left(\overleftarrow{\tilde{q}}_{k, i}(t)\right) \overleftarrow{\dot{q}}_{k, i}(t) \\
& \delta_{e}^{\left[\mathbf{x}_{h}\right]}\left(t, t_{e}^{\left[\mathbf{x}_{h}\right]}\right)=\hat{W}_{e}^{\left[\mathbf{x}_{h}\right]^{T}}\left(t_{e}^{\left[\mathbf{x}_{h}\right]}\right) \omega_{e}(t)+L\left(\overleftarrow{q}_{k, i}(t), u_{ \pm}(t)\right) \\
& \lambda_{e}^{\left[\mathbf{x}_{h}\right]}\left(t, t_{e}^{\left[\mathbf{x}_{h}\right]}\right)=k_{\lambda}\left(1-e^{-\left|\delta_{e}^{\left[\mathbf{x}_{h}\right]}\left(t, t_{e}^{\left[\mathbf{x}_{h}\right]}\right)\right|}\right),
\end{aligned}
$$

where $k_{\lambda} \in \mathbb{R}^{+}$is a positive tuning parameter, $\delta_{e}^{\left[\mathbf{x}_{h}\right]}$ is the Bellman error, and $\lambda_{e}^{\left[\mathbf{x}_{h}\right]}$ is the forgetting factor [37]. When $\overleftarrow{q}_{k, i}(t) \in\left[\mathbf{x}_{h}\right]$, the critic is designed as follows:

$$
\begin{aligned}
\dot{\hat{W}}_{e}^{\left[\mathbf{x}_{h}\right]}\left(t_{e}^{\left[\mathbf{x}_{h}\right]}\right)= & -k_{c} \Gamma_{e}^{\left[\mathbf{x}_{h}\right]}\left(t_{e}^{\left[\mathbf{x}_{h}\right]}\right) \\
& \times \frac{\omega_{e}(t)}{1+\omega_{e}(t)^{T} \Gamma_{e}^{\left[\mathbf{x}_{h}\right]}\left(t_{e}^{\left[\mathbf{x}_{h}\right]}\right) \omega_{e}(t)} \delta_{e}^{\left[\mathbf{x}_{h}\right]}\left(t, t_{e}^{\left[\mathbf{x}_{h}\right]}\right) \\
\dot{\Gamma}_{e}^{\left[\mathbf{x}_{h}\right]}\left(t_{e}^{\left[\mathbf{x}_{h}\right]}\right)= & -k_{c}\left(-\lambda_{e}^{\left[\mathbf{x}_{h}\right]}\left(t, t_{e}^{\left[\mathbf{x}_{h}\right]}\right) \Gamma_{e}^{\left[\mathbf{x}_{h}\right]}\left(t_{e}^{\left[\mathbf{x}_{h}\right]}\right)+\Gamma_{e}^{\left[\mathbf{x}_{h}\right]}\left(t_{e}^{\left[\mathbf{x}_{h}\right]}\right)\right. \\
& \left.\times \frac{\omega_{e}(t) \omega_{e}(t)^{T}}{1+\omega_{e}(t)^{T} \Gamma_{e}^{\left[\mathbf{x}_{h}\right]}\left(t_{e}^{\left[\mathbf{x}_{h}\right]}\right) \omega_{e}(t)} \Gamma_{e}^{\left[\mathbf{x}_{h}\right]}\left(t_{e}^{\left[\mathbf{x}_{h}\right]}\right)\right)
\end{aligned}
$$


where $k_{c} \in \mathbb{R}^{+}$is a positive tuning parameter. We denote the dynamic systems in (41) as $F_{c}$. Following the above derivations, we can propose the theorem infra:

Theorem 4: Assume $\frac{\omega_{e}}{\sqrt{1+\omega_{e}^{T} \Gamma_{e}^{\left[\mathbf{x}_{h}\right]} \omega_{e}}}$ is persistently exciting. By adopting the critic shown in (41), the estimation error $\| \tilde{W}_{e}^{\left[\mathbf{x}_{h}\right] \|=}$ $\left\|W_{e}^{\left[\mathbf{x}_{h}\right]}-\hat{W}_{e}^{\left[\mathbf{x}_{h}\right]}\right\|$ is uniformly ultimately bounded (UUB). If the neural network (NN) in (38b) is ideal and $u_{ \pm}$is generated by backward propagation using PMP, $\hat{W}_{e}^{\left[\mathbf{x}_{h}\right]}$ exponentially and asymptotically converges to the HJB solution to the game described in (8) and (10).

Proof: For simplicity, we denote $u_{ \pm}=u_{ \pm}\left(e_{(i, j)}, W_{e}^{\left[\mathbf{x}_{h}\right]}\right) ; u_{ \pm}^{\star}=$ $u_{ \pm}^{\star}\left(e_{(i, j)}, W_{e}^{\left[\mathbf{x}_{h}\right]}\right)$, where $u_{ \pm}^{\star}$ is the Pareto optimum to the game described in (8) and (10); $\tilde{u}_{ \pm}=u_{ \pm}^{\star}-u_{ \pm}$. The local timer $t_{e}^{\left[\mathbf{x}_{h}\right]}$ is omitted in the subsequent context for simplicity. Given (38c) and (40a), we can derive

$$
\begin{aligned}
0= & W_{e}^{\left[\mathbf{x}_{h}\right]^{T}} \omega_{e}\left(\overleftarrow{q}_{k, i}, u_{ \pm}^{\star}\right)+L\left(\overleftarrow{q}_{k, i}, u_{ \pm}^{\star}\right) \\
& +\nabla \varepsilon_{e}^{\left[\mathbf{x}_{h}\right]}\left(\overleftarrow{\tilde{q}}_{k, i}\right)\left(f_{e}\left(\overleftarrow{q}_{k, i}\right)+g_{e}^{ \pm}\left(\overleftarrow{q}_{k, i}\right) u_{ \pm}^{\star}\right)
\end{aligned}
$$

Then, denoting $\delta_{e}^{\left[\mathbf{x}_{h}\right]}=\delta_{e}^{\left[\mathbf{x}_{h}\right]}\left(\hat{W}_{e}^{\left[\mathbf{x}_{h}\right]}, W_{e}^{\left[\mathbf{x}_{h}\right]}, \overleftarrow{q}{ }_{k, i}, u_{ \pm}, u_{ \pm}^{\star}\right)$ and $\omega_{e}=\omega_{e}\left(\overleftarrow{q}_{k, i}, u_{ \pm}\right)$, (40b) can be rewritten as

$$
\begin{aligned}
\delta_{e}^{\left[\mathbf{x}_{h}\right]}= & -\tilde{W}_{e}^{\left[\mathbf{x}_{h}\right]^{T}} \omega_{e}\left(\overleftarrow{q}_{k, i}, u_{ \pm}\right)-\tilde{u}_{ \pm}^{T} R_{2}\left(u_{ \pm}^{\star}+u_{ \pm}\right) \\
& +\nabla \varepsilon_{e}^{\left[\mathbf{x}_{h}\right]}\left(\overleftarrow{\tilde{q}}_{k, i}\right)\left(f_{e}\left(\overleftarrow{q}_{k, i}\right)+g_{e}^{ \pm}\left(\overleftarrow{q}_{k, i}\right) u_{ \pm}^{\star}\right) \\
& -W_{e}^{\left[\mathbf{x}_{h}\right]^{T}} \nabla \sigma\left(\overleftarrow{\tilde{q}}_{k, i}\right) g_{e}^{ \pm}\left(\overleftarrow{q}_{k, i}\right) \tilde{u}_{ \pm} .
\end{aligned}
$$

Given (43) and a Lyapunov function $\mathcal{V}_{e}^{\left[\mathbf{x}_{h}\right]}\left(\tilde{W}_{e}^{\left[\mathbf{x}_{h}\right]}\right)=$ $\tilde{W}_{e}^{\left[\mathbf{x}_{h}\right]^{T}} \Gamma_{e}^{\left[\mathbf{x}_{h}\right]^{-1}} \tilde{W}_{e}^{\left[\mathbf{x}_{h}\right]}$, we have

$$
\begin{aligned}
& \dot{\mathcal{V}}_{e}^{\left[\mathbf{x}_{h}\right]}=\dot{\tilde{W}}_{e}^{\left[\mathbf{x}_{h}\right]^{T}} \Gamma_{e}^{\left[\mathbf{x}_{h}\right]^{-1}} \tilde{W}_{e}^{\left[\mathbf{x}_{h}\right]}+\tilde{W}_{e}^{\left[\mathbf{x}_{h}\right]^{T}} \frac{d}{d t}\left(\Gamma_{e}^{\left[\mathbf{x}_{h}\right]^{-1}} \tilde{W}_{e}^{\left[\mathbf{x}_{h}\right]}\right) \\
& =2 \tilde{W}_{e}^{\left[\mathbf{x}_{h}\right]^{T}} \Gamma_{e}^{\left[\mathbf{x}_{h}\right]^{-1}} \dot{\tilde{W}}_{e}^{\left[\mathbf{x}_{h}\right]}+\tilde{W}_{e}^{\left[\mathbf{x}_{h}\right]^{T}} \frac{d \Gamma_{e}^{\left[\mathbf{x}_{h}\right]^{-1}}}{d t} \tilde{W}_{e}^{\left[\mathbf{x}_{h}\right]} \\
& =-k_{c} \frac{\tilde{W}_{e}^{\left[\mathbf{x}_{h}\right]^{T}} \omega_{e} \omega_{e}^{T} \tilde{W}_{e}^{\left[\mathbf{x}_{h}\right]}}{1+\omega_{e}^{T} \Gamma_{e}^{\left[\mathbf{x}_{h}\right]} \omega_{e}} \\
& -k_{c} k_{\lambda}\left(1-e^{-\left|\delta_{e}^{\left[\mathbf{x}_{h}\right]}\right|}\right) \tilde{W}_{e}^{\left[\mathbf{x}_{h}\right]^{T}} \Gamma_{e}^{\left[\mathbf{x}_{h}\right]^{-1}} \tilde{W}_{e}^{\left[\mathbf{x}_{h}\right]} \\
& +2 k_{c} \frac{\tilde{W}_{e}^{\left[\mathbf{x}_{h}\right]^{T}} \omega_{e}}{1+\omega_{e}^{T} \Gamma_{e}^{\left[\mathbf{x}_{h}\right]} \omega_{e}}\left(-\tilde{u}_{ \pm}^{T} R_{2}\left(u_{ \pm}^{\star}+u_{ \pm}\right)\right. \\
& +\nabla \varepsilon_{e}^{\left[\mathbf{x}_{h}\right]}\left(\overleftarrow{\tilde{q}}_{k, i}\right)\left(f_{e}\left(\overleftarrow{q}_{k, i}\right)+g_{e}^{ \pm}\left(\overleftarrow{q}_{k, i}\right) u_{ \pm}^{\star}\right)
\end{aligned}
$$

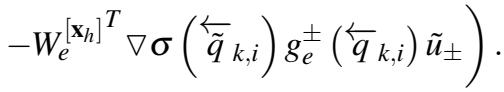

As $\sup _{\star}\left\|u_{ \pm}^{\star}\right\| \leq \varsigma_{u}$, sup $\left\|u_{ \pm}\right\| \leq \varsigma_{u}$ and Assumption 3 hold, $\left\|\tilde{u}_{ \pm}\right\|$ $u_{ \pm}^{\star} \in\left[U_{2}\right]$

and $\left\|f_{e}\left(\overleftarrow{q}_{k, i}\right)+g_{e}^{ \pm}\left(\overleftarrow{q}_{k, i}\right) u_{ \pm}^{\star}\right\|$ are bounded. Moreover, given that $\sup _{\overleftarrow{q}_{k, i} \in\left[\mathbf{x}_{h}\right]}\left\|\nabla \varepsilon_{e}^{\left[\mathbf{x}_{h}\right]}\left(\overleftarrow{\tilde{q}}_{k, i}\right)\right\| \leq \varsigma_{\nabla \varepsilon}^{\left[\mathbf{x}_{h}\right]}$, then $\nabla \varepsilon_{e}^{\left[\mathbf{x}_{h}\right]}\left(\overleftarrow{\tilde{q}}_{k, i}\right) \overleftarrow{q}_{k, i}$ is bounded. Provided that $\frac{\omega_{e}}{\sqrt{1+\omega_{e}^{T} \Gamma_{e}^{\left[\mathbf{x}_{h}\right]} \omega_{e}}}$ is persistently exciting, therefore, $\left\|\Gamma_{e}^{\left[\mathbf{x}_{h}\right]}\right\|$ is bounded [37]. Thus, there exists a bound $\varsigma_{\tilde{W}_{e}^{[}}\left[\mathbf{x}_{h}\right]$, when
$\varsigma_{\tilde{W}_{e}^{\left[\mathbf{x}_{h}\right]}} \leq\left\|\tilde{W}_{e}^{\left[\mathbf{x}_{h}\right]}\right\|, \dot{\mathcal{V}}_{e}^{\left[\mathbf{x}_{h}\right]}\left(\tilde{W}_{e}^{\left[\mathbf{x}_{h}\right]}\right)<0$.

If the NN structure is ideal, then $\sup _{\overleftarrow{q}_{k, i} \in\left[\mathbf{x}_{h}\right]}\left\|\nabla \varepsilon_{e}^{\left[\mathbf{x}_{h}\right]}\left(\overleftarrow{\tilde{q}}_{k, i}\right)\right\|=0$. Provided that both $\left[U_{2}\right]$ and $J\left(e_{(i, j)}\right)$ are convex and $u_{ \pm}$is computed from (35), the sufficient condition is satisfied for ensuring the optimality by PMP. Thus, when $u_{ \pm}=u_{ \pm}^{\star}$, then $\dot{\mathcal{V}}_{e}^{\left[\mathbf{x}_{h}\right]}<0$ always holds.

When the backward propagation for one iteration has been completed, the subpavings $\mathbb{D}$ and $\partial \mathbb{D}$ are updated as follows:

$$
\begin{aligned}
\partial \mathbb{D}_{k} & \left.=\cup\left\{[\mathbf{x}] \subset \overline{\mathbb{K}} \mid[\mathbf{x}] \backslash \partial[\mathbf{x}] \cap \partial \mathcal{D}_{k} \neq \varnothing\right]\right\}, \\
\underline{D}_{k} & =\cup\left\{[\mathbf{x}] \subset \overline{\mathbb{K}} \mid[\mathbf{x}] \subset \mathcal{D}_{k}\right\} .
\end{aligned}
$$

Moreover, we remove and interpolate points to ensure that every box $\left[\mathbf{x}_{h}\right] \subset \partial \mathbb{D}_{k}$ contains one point. The interpolated states $\hat{\phi}_{k, i}$ can be estimated as follows:

$$
\begin{aligned}
& \hat{q}_{k, i}:\left[\mathbf{x}_{h}\right] \subset \partial \mathbb{D}_{k}, \quad \hat{q}_{k, i} \in\left[\mathbf{x}_{h}\right] \backslash \partial\left[\mathbf{x}_{h}\right] \cap \partial \mathcal{D}_{k},
\end{aligned}
$$

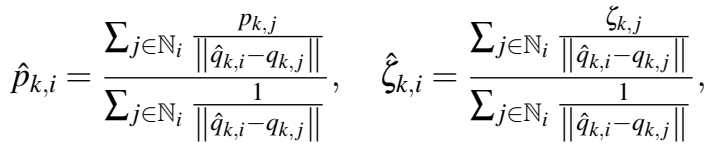

where $q_{k, j}: j \in \mathbb{N}_{i}$ stands for the vertices that share the common segments of $\mathcal{D}_{k}$ with $q_{k, i}$, and $\mathbb{N}_{i}$ is the set of their indexes.

The pseudo code in Algorithm 1 shows the detailed steps to perform the off-line backward propagation.

\section{B. Cooperative Learning}

The on-line reinforcement learning process is taken under the following assumption:

Assumption 5: $\forall t \geq 0$, the communication graph $\mathcal{G}_{N}$ is always connected, and

$$
\begin{aligned}
\forall i, j \in[1, N], \quad i \neq j, \quad e_{(i, j)}(t) & \in \underline{\operatorname{Capt}_{\tilde{F}_{e}}(X, C)} \\
\wedge \forall e_{(i, j)}(t) \notin \underline{\operatorname{Capt}_{\tilde{F}_{e}}(K, C),}, \quad \alpha_{i j} & =0,
\end{aligned}
$$

such that there always exists a distributed control strategy for the network system to reach consensus.

We assign a critic and an actor for each local network system, where the critic learns the local value function and the actor learns the optimal control policy. Regarding the $i$ th vehicle $v_{i}$, the parameters of its critic and actor are partially shared by the critics and actors of its neighbouring vehicles $v_{\mathcal{N}_{i}}$, respectively. Denoting $(\cdot)_{\mathcal{N}_{i}}=\left\{(\cdot)_{(i, j)}\right\},(\cdot)_{(i, j)}$ is shared by the $i$ th and the $j$ th critic (or actor).

The designed critic in cooperative learning aims to update $\hat{\mathcal{W}}_{\mathcal{N}_{i}}^{c}=\left[\cdots, \hat{\mathcal{W}}_{(i, j)}^{\left[\mathbf{x}_{h}\right]^{T}}, \cdots\right]^{T}: j \in \mathcal{N}_{i}$, when $\alpha_{i j}>0 \wedge h=$ $\rho\left(e_{(i, j)}(t)\right) \wedge\left[\mathbf{x}_{h}\right] \subset \overline{\operatorname{Capt}_{\tilde{F}_{e}}(K, C)} \backslash \mathbb{C}$. The critic is composed of two independent parts, and they are updated simultaneously. The first part aims to approximate $W_{e}^{\left[\mathbf{x}_{h}\right]}$ as shown in (38c). The parameters $(\cdot)_{e}^{\left[\mathbf{x}_{h}\right]}$ in Section IV-A are shared throughout the whole graph. These parameters, together with their timer $t_{e}^{\left[\mathbf{x}_{h}\right]}$, are updated via (40) and (41) with slight modifications:

$$
\begin{aligned}
\omega_{(i, j)} & =\nabla \sigma\left(\tilde{e}_{(i, j)}\right)\left(f_{e}\left(e_{(i, j)}\right)+g_{e}^{ \pm}\left(e_{(i, j)}\right) u_{ \pm}\right), \\
u_{ \pm} & =\underset{u_{i j} \in\left[U_{2}\right]}{\arg \min }\left\{\hat{W}_{e}^{\left[\mathbf{x}_{h}\right]^{T}} \nabla \sigma\left(\tilde{e}_{(i, j)}\right) g_{e}^{ \pm}\left(e_{(i, j)}\right) u_{i j}+u_{i j}^{T} R_{2} u_{i j}\right\},
\end{aligned}
$$

$$
\tilde{e}_{(i, j)}=e_{(i, j)}-\operatorname{mid}\left(\left[\mathbf{x}_{h}\right]\right)
$$




Algorithm 1 Computation of an inner approximation of
Capt $_{\tilde{F}_{e}}(K, C)$.

Generate grids and subpavings $\mathbb{C}, \overline{\mathbb{C}}, \mathbb{K}, \overline{\mathbb{K}}$;

$\underline{\mathbb{D}}_{0}=\mathbb{C} ; \partial \mathbb{D}_{0}=\partial \mathbb{C} ; k=0 ; t=0 ;$

Allocate vertices $\mathbf{Q}_{0}$ such that $\overleftarrow{q}_{0, i}(0) \in\left[\mathbf{x}_{0, i}\right] \backslash \partial\left[\mathbf{x}_{0, i}\right] \cap \partial C$;

$\overleftarrow{p}_{0, i}(0)=\nabla \psi_{C}\left(\overleftarrow{q}_{0, i}(0)\right) ; \overleftarrow{\zeta}_{0, i}(0)=0$

while $\partial \mathcal{D}_{k+1} \neq \partial \mathcal{D}_{k}$ do

$I_{k}=\operatorname{size}\left(\partial \mathbb{D}_{k}\right) ; \triangle t_{k}=0$

while $\Delta t_{k}=0$ satisfies (37b) do

for $i=1 ; i \leq I_{k} ; i++$ do

$u_{ \pm}$is calculated by (35);

if Condition 1 holds true then

Set $\triangle \tau_{k} \ll \Delta t_{k}$;

$\phi_{k, i}=\varphi_{\overleftarrow{F}_{\phi}}\left(\triangle \tau_{k} ; \phi_{k, i}, u_{ \pm}\right) ; h=\rho\left(\overleftarrow{q}_{k, i}\right)$

if $\overleftarrow{q}_{k, i}$ is the first point that enters $\left[\mathbf{x}_{h}\right]$ then $\hat{W}_{e}^{\left[\mathbf{x}_{h}\right]}$ and $\omega_{e}$ are initialized by (39);

else

$\hat{W}_{e}^{\left[\mathbf{x}_{h}\right]}=\varphi_{F_{c}}\left(\triangle t_{k} ; \hat{W}_{e}^{\left[\mathbf{x}_{h}\right]}, u_{ \pm}\right) ;$

$\Gamma_{e}^{\left[\mathbf{x}_{h}\right]}=\varphi_{F_{c}}\left(\triangle t_{k} ; \Gamma_{e}^{\left[\mathbf{x}_{h}\right]}, u_{ \pm}\right)$;

end if

end if

end for

$\triangle t_{k}=\Delta t_{k}+\Delta \tau_{k}$

\section{end while}

$k=k+1 ; t=t+\Delta t_{k} ; \partial \mathbb{D}_{k}$ and $\underline{\mathbb{D}}_{k}$ are updated by (45);

Adjust $\mathbf{Q}_{k}$ by removing and interpolating states such that

$\forall[\mathbf{x}] \subset \partial \mathbb{D}_{k}$ contains only one point $q$; the interpolated states are estimated as shown in (46)

\section{end while}

$T=t ; \operatorname{Capt}_{\tilde{F}_{e}}(K, C)=\underline{D}_{k}$;

The second part aims to approximate the instantaneous weight $\beta_{(i, j)} \in \mathbb{R}^{+}$on $W_{e}^{\left[\mathbf{x}_{h}\right]}$. The value functions for local network systems can be constructed by NN as follows:

$$
V_{\mathcal{N}_{i}}\left(e_{\mathcal{N}_{i}}\right)=\sum_{j \in \mathcal{N}_{i}} \mathcal{W}_{(i, j)}^{\left[\mathbf{x}_{h}\right]}{ }^{T} \sigma\left(\tilde{e}_{(i, j)}\right)+\sum_{j \in \mathcal{N}_{i}} \varepsilon_{(i, j)}^{\left[\mathbf{x}_{h}\right]}\left(\tilde{e}_{(i, j)}\right)
$$

where $h=\rho\left(e_{(i, j)}\right): e_{(i, j)} \in\left[\mathbf{x}_{h}\right] ; \mathcal{W}_{(i, j)}^{\left[\mathbf{x}_{h}\right]}$ stands for the true $\mathrm{NN}$ weight and $\mathcal{W}_{(i, j)}^{\left[\mathbf{x}_{h}\right]}(t)=\beta_{(i, j)}(t) W_{e}^{\left[\mathbf{x}_{h}\right]} ; \varepsilon_{(i, j)}^{\left[\mathbf{x}_{h}\right]}\left(t, \tilde{e}_{(i, j)}(t)\right)=$ $\beta_{(i, j)}(t) \varepsilon_{e}^{\left[\mathbf{x}_{h}\right]}\left(\tilde{e}_{(i, j)}(t)\right) ; \quad \sup _{e_{(i, j)} \in\left[\mathbf{x}_{h}\right]}\left\|\varepsilon_{e}^{\left[\mathbf{x}_{h}\right]}\left(\tilde{e}_{(i, j)}\right)\right\| \leq \varsigma_{\varepsilon}^{\left[\mathbf{x}_{h}\right]} ; \quad$ and $\left.\sup _{(i, j)} \| \nabla \mathbf{x}_{h}\right]\left[\varepsilon_{e}^{\left[\mathbf{x}_{h}\right]}\left(\tilde{e}_{(i, j)}\right) \| \leq \varsigma_{\nabla \varepsilon}^{\left[\mathbf{x}_{h}\right]}\right.$. The estimated value functions $\hat{V}_{\mathcal{N}_{i}}$ can be written as

$$
\hat{V}_{\mathcal{N}_{i}}\left(e_{\mathcal{N}_{i}}\right)=\sum_{j \in \mathcal{N}_{i}} \hat{\mathcal{W}}_{(i, j)}^{\left[\mathbf{x}_{h}\right]^{T}} \sigma\left(\tilde{e}_{(i, j)}\right)
$$

where $\hat{\mathcal{W}}_{(i, j)}^{\left[\mathbf{x}_{h}\right]}(t)=\hat{\beta}_{(i, j)}(t) \hat{W}_{e}^{\left[\mathbf{x}_{h}\right]}$ and $\hat{\beta}_{(i, j)}$ is the estimated dynamic weight. Before the on-line reinforcement training, the parameters of critics for all the relative error systems are initialized as follows: for $\forall \alpha_{i j}>0, \hat{\mathcal{W}}_{(i, j)}^{\left[\mathbf{x}_{h}\right]}(0)=\hat{\beta}_{(i, j)}(0) \hat{W}_{e}^{\left[\mathbf{x}_{h}\right]}$ when $\left[\mathbf{x}_{h}\right] \subset$ $\overline{\operatorname{Capt}_{\tilde{F}_{e}}(K, C)} \backslash \mathbb{C}$, and $\hat{\mathcal{W}}_{(i, j)}^{\left[\mathbf{x}_{h}\right]}(0)=\mathbf{0}$ when $\left[\mathbf{x}_{h}\right] \subset \mathbb{C}$.

Let $\quad \tilde{e}_{\mathcal{N}_{i}}=\left[\cdots, \tilde{e}_{(i, j)}^{T}, \cdots\right]^{T}: j \in \mathcal{N}_{i}, \quad \nabla \sigma_{\mathcal{N}_{i}}\left(\tilde{e}_{\mathcal{N}_{i}}\right)=$ $\operatorname{diag}\left(\left[\cdots, \nabla \sigma\left(\tilde{e}_{(i, j)}\right), \cdots\right]\right): j \in \mathcal{N}_{i}$, and for the other variables, let $(\cdot)_{\mathcal{N}_{i}}=\left[\cdots,(\cdot)_{(i, j)}{ }^{T}, \cdots\right]^{T}: j \in \mathcal{N}_{i}$, for $i \in[1, N], j \in \mathcal{N}_{i}, i \neq j$ and $\left[\mathbf{x}_{h}\right] \subset \operatorname{Capt}_{\tilde{F}_{e}}(K, C) \backslash \mathbb{C}$, the estimated dynamic weight $\hat{\beta}_{(i, j)}(t)$ is updated as shown below:

$$
\begin{aligned}
\dot{\hat{\beta}}_{(i, j)}= & -k_{\beta} \hat{W}_{e}^{\left[\mathbf{x}_{h}\right]^{T}} \nabla \sigma\left(\tilde{e}_{(i, j)}\right)\left(\delta_{\mathcal{N}_{i}}\left(\frac{1}{2} f_{e}\left(e_{(i, j)}\right)+g_{e}^{+}\left(e_{(i, j)}\right) u_{i}\right)\right. \\
& \left.+\delta_{\mathcal{N}_{j}}\left(\frac{1}{2} f_{e}\left(e_{(j, i)}\right)+g_{e}^{+}\left(e_{(j, i)}\right) u_{j}\right)\right)
\end{aligned}
$$

where $k_{\beta}$ is a positive tuning parameter, $\hat{\beta}_{(i, j)}(t)$ is constrained to $[0, \infty)$ and $\hat{\beta}_{(i, j)}(0)=1 ; \delta_{\mathcal{N}_{i}}$ is the Bellman error corresponding to the local Hamiltonian $H_{\mathcal{N}_{i}}$ in (19), which is defined as

$$
\begin{aligned}
\delta_{\mathcal{N}_{i}}= & \hat{\mathcal{W}}_{\mathcal{N}_{i}}^{c}{ }^{T} \nabla \sigma_{\mathcal{N}_{i}}\left(\tilde{e}_{\mathcal{N}_{i}}\right) g_{\mathcal{N}_{i}}^{+}\left(e_{\mathcal{N}_{i}}\right) u_{i}+\frac{1}{2} \hat{\mathcal{W}}_{\mathcal{N}_{i}}^{c}{ }^{T} \nabla \sigma_{\mathcal{N}_{i}}\left(\tilde{e}_{\mathcal{N}_{i}}\right) f_{e_{\mathcal{N}}}\left(e_{\mathcal{N}_{i}}\right) \\
& +\left|\mathcal{N}_{i}\right| u_{i}^{T} R u_{i}+\frac{1}{2} \sum_{j \in \mathcal{N}_{i}} Q\left(e_{(i, j)}\right)
\end{aligned}
$$

and in this way, the $i$ th and the $j$ th critics update their common parameter $\hat{\beta}_{(i, j)}$ to minimize both $\left|\delta_{\mathcal{N}_{i}}\right|$ and $\left|\delta_{\mathcal{N}_{j}}\right|$.

The updating law for the actor is designed below, for $j \in \mathcal{N}_{i}$ and $i \in[1, N]$ :

$$
\begin{aligned}
\dot{\hat{S}}_{(i, j)}^{a} & =\frac{k_{c} k_{a}\left|\delta_{e}^{\left[\mathbf{x}_{h}\right]}\right|\left\|\Gamma_{e}^{\left[\mathbf{x}_{h}\right]} \omega_{(i, j)}\right\|\left(\hat{W}_{e}^{\left[\mathbf{x}_{h}\right]}-\hat{S}_{(i, j)}^{a}\right)}{\left(\lambda_{e}^{\left[\mathbf{x}_{h}\right]}+\varepsilon_{a}\right)\left|1+\omega_{(i, j)}{ }^{T} \Gamma_{e}^{\left[\mathbf{x}_{h}\right]} \omega_{(i, j)}\right|}, \\
\hat{\mathcal{W}}_{(i, j)}^{a} & =\hat{\beta}_{(i, j)} \hat{S}_{(i, j)}^{a},
\end{aligned}
$$

where $S \in \mathbb{R}^{l} ; \hat{S}_{(i, j)}^{a}(0)=\hat{W}_{e}^{\left[\mathbf{x}_{h}\right]} ; \varepsilon_{a} \in \mathbb{R}^{+}$is a small positive constant and $k_{a} \in \mathbb{R}^{+}$is a positive tuning parameter.

The distributed control policy for the $i$ th vehicle $v_{i}$ is designed as below

$$
\begin{aligned}
u_{i}\left(e_{\mathcal{N}_{i}}, \hat{\mathcal{W}}_{\mathcal{N}_{i}}^{a}\right)=\underset{u_{i} \in[U]}{\arg \min }\{ & \left|\mathcal{N}_{i}\right| u_{i}^{T} R u_{i} \\
& \left.+\hat{\mathcal{W}}_{\mathcal{N}_{i}}^{a}{ }^{T} \nabla \sigma_{\mathcal{N}_{i}}\left(\tilde{e}_{\mathcal{N}_{i}}\right) g_{\mathcal{N}_{i}}^{+}\left(e_{\mathcal{N}_{i}}\right) u_{i}\right\},
\end{aligned}
$$

and if $\forall i, j \in[1, N], e_{(i, j)} \in \partial C$, then $\hat{W}_{e}^{\left[\mathbf{x}_{h}\right]}=\nabla \psi_{C}\left(e_{(i, j)}\right)$. Denote $\mathcal{W}_{\mathcal{N}_{i}}=\left[\cdots, \mathcal{W}_{(i, j)}^{\left[\mathbf{x}_{h}\right]^{T}}, \cdots\right]^{T}: j \in \mathcal{N}_{i}$, given (18) and (49), the Pareto optimal distributed control policy can be written as

$$
\begin{aligned}
u_{i}^{\star}\left(e_{\mathcal{N}_{i}}, \mathcal{W}_{\mathcal{N}_{i}}\right)=\underset{u_{i} \in[U]}{\arg \min }\{ & \mathcal{W}_{\mathcal{N}_{i}}^{T} \nabla \sigma_{\mathcal{N}_{i}}\left(\tilde{e}_{\mathcal{N}_{i}}\right) g_{\mathcal{N}_{i}}^{+}\left(e_{\mathcal{N}_{i}}\right) u_{i} \\
& \left.+\left|\mathcal{N}_{i}\right| u_{i}^{T} R u_{i}+\nabla \varepsilon_{\mathcal{N}_{i}}\left(\tilde{e}_{\mathcal{N}_{i}}\right) g_{\mathcal{N}_{i}}^{+}\left(e_{\mathcal{N}_{i}}\right) u_{i}\right\} .
\end{aligned}
$$

\section{Convergence Analysis}

The estimation errors on the NN weights of the $i$ th critic and the $i$ th actor are derived by $\tilde{\mathcal{W}}_{\mathcal{N}_{i}}^{c}=\mathcal{W}_{\mathcal{N}_{i}}-\hat{\mathcal{W}}_{\mathcal{N}_{i}}^{c}$ and $\tilde{\mathcal{W}}_{\mathcal{N}_{i}}^{a}=\mathcal{W}_{\mathcal{N}_{i}}-\hat{\mathcal{W}}_{\mathcal{N}_{i}}^{a}$, respectively. Their elements are expressed as $\tilde{\mathcal{W}}_{(i, j)}^{\left[\mathbf{x}_{h}\right]}(t)=\beta_{(i, j)}(t) W_{e}^{\left[\mathbf{x}_{h}\right]}-\hat{\beta}_{(i, j)}(t) \hat{W}_{e}^{\left[\mathbf{x}_{h}\right]}$ and $\tilde{\mathcal{W}}_{(i, j)}^{a}(t)=$ $\beta_{(i, j)}(t) W_{e}^{\left[\mathbf{x}_{h}\right]}-\hat{\beta}_{(i, j)}(t) \hat{S}_{(i, j)}^{a}$, respectively. Moreover, the control error is given by $\tilde{u}_{i}=u_{i}^{\star}-u_{i}$ where $\left\|\tilde{u}_{i}\right\| \leq \varsigma_{\tilde{u}_{i}}$ when $t \rightarrow \infty$.

Theorem 5: Assume $\frac{\omega_{(i, j)}}{\sqrt{1+\omega_{(i, j)} \Gamma_{e}^{\left[\mathbf{x}_{h}\right]} \omega_{(i, j)}}}$ is persistently exciting. From the local critic given (41) and (51) as well as the local actor 


$$
\begin{aligned}
& \dot{\mathcal{V}}_{\beta}=-\sum_{i, j=1}^{N} \alpha_{i j} \tilde{\beta}_{(i, j)} \hat{W}_{e}^{\left[\mathbf{x}_{h}\right]^{T}} \nabla \sigma\left(\tilde{e}_{(i, j)}\right) \\
& \times\left(\left(\frac{1}{2} f_{e}\left(e_{(i, j)}\right)+g_{e}^{+}\left(e_{(i, j)}\right) u_{i}\right)\left(\left(\frac{1}{2} f_{e_{\mathcal{N}}}\left(e_{\mathcal{N}_{i}}\right)+g_{\mathcal{N}_{i}}^{+}\left(e_{\mathcal{N}_{i}}\right) u_{i}\right)^{T} \nabla \sigma_{\mathcal{N}_{i}}^{T}\left(\tilde{e}_{\mathcal{N}_{i}}\right) \tilde{\beta}_{\mathcal{N}_{i}} \otimes I_{l} \hat{W}_{\mathcal{N}_{i}}^{e}+\aleph_{\beta_{i}}+\aleph_{W_{i}}+\aleph_{\varepsilon_{i}}+\aleph_{R_{i}}\right)\right. \\
& \left.+\left(\frac{1}{2} f_{e}\left(e_{(j, i)}\right)+g_{e}^{+}\left(e_{(j, i)}\right) u_{j}\right)\left(\left(\frac{1}{2} f_{e_{\mathcal{N}}}\left(e_{\mathcal{N}_{j}}\right)+g_{\mathcal{N}_{j}}^{+}\left(e_{\mathcal{N}_{j}}\right) u_{j}\right)^{T} \nabla \sigma_{\mathcal{N}_{j}}^{T}\left(\tilde{e}_{\mathcal{N}_{j}}\right) \tilde{\beta}_{\mathcal{N}_{j}} \otimes I_{l} \hat{W}_{\mathcal{N}_{j}}^{e}+\aleph_{\beta_{j}}+\aleph_{W_{j}}+\aleph_{\varepsilon_{j}}+\aleph_{R_{j}}\right)\right) .
\end{aligned}
$$

formulated in (53), $\forall i \in[1, N], j \in \mathcal{N}_{i},\left\|\tilde{\mathcal{W}}_{\mathcal{N}_{i}}^{c}\right\|$ and $\left\|\tilde{\mathcal{W}}_{\mathcal{N}_{i}}^{a}\right\|$ are UUB. If the NN structure and the tuning parameters are ideal, and the state resolution $\varepsilon_{X}$ in backward propagation is small enough, then $\varsigma_{\tilde{u}_{i}}$ can be arbitrarily small such that $u_{i}$ can converge to the Pareto optimal strategy $u_{i}^{\star}$ of the game described in (13) and (14).

Proof: For simplicity, denote $u_{i}^{\star}=u_{i}^{\star}\left(e_{\mathcal{N}_{i}}, \mathcal{W}_{\mathcal{N}_{i}}\right), u_{i}=$ $u_{i}\left(e_{\mathcal{N}_{i}}, \hat{\mathcal{W}}_{\mathcal{N}_{i}}^{a}\right)$. For the true NN weight $\mathcal{W}_{\mathcal{N}_{i}}$, the following property always holds:

$$
\begin{aligned}
0= & \frac{1}{2} \mathcal{W}_{\mathcal{N}_{i}}^{T} \nabla \sigma_{\mathcal{N}_{i}}\left(\tilde{e}_{\mathcal{N}_{i}}\right) f_{e_{\mathcal{N}}}\left(e_{\mathcal{N}_{i}}\right)+\mathcal{W}_{\mathcal{N}_{i}}^{T} \nabla \sigma_{\mathcal{N}_{i}}\left(\tilde{e}_{\mathcal{N}_{i}}\right) g_{\mathcal{N}_{i}}^{+}\left(e_{\mathcal{N}_{i}}\right) u_{i}^{\star} \\
& +\frac{1}{2} \nabla \varepsilon_{\mathcal{N}_{i}}\left(\tilde{e}_{\mathcal{N}_{i}}\right) f_{e_{\mathcal{N}}}\left(e_{\mathcal{N}_{i}}\right)+\nabla \varepsilon_{\mathcal{N}_{i}}\left(\tilde{e}_{\mathcal{N}_{i}}\right) g_{\mathcal{N}_{i}}^{+} u_{i}^{\star} \\
& +\left|\mathcal{N}_{i}\right| u_{i}^{\star T} R u_{i}^{\star}+\frac{1}{2} \sum_{j \in \mathcal{N}_{i}} Q\left(e_{(i, j)}\right)
\end{aligned}
$$

and given (56), by denoting $\delta_{\mathcal{N}_{i}}=\delta_{\mathcal{N}_{i}}\left(\hat{\mathcal{W}}_{\mathcal{N}_{i}}^{c}, \mathcal{W}_{\mathcal{N}_{i}}, e_{\mathcal{N}_{i}}, u_{i}, u_{i}^{\star}\right)$, (52) can be recast to

$$
\begin{aligned}
& \delta_{\mathcal{N}_{i}}=\hat{\mathcal{W}}_{\mathcal{N}_{i}}^{c}{ }^{T} \nabla \sigma_{\mathcal{N}_{i}}\left(\tilde{e}_{\mathcal{N}_{i}}\right) g_{\mathcal{N}_{i}}^{+}\left(e_{\mathcal{N}_{i}}\right) u_{i}+\frac{1}{2} \hat{\mathcal{W}}_{\mathcal{N}_{i}}^{c}{ }^{T} \nabla \sigma_{\mathcal{N}_{i}}\left(\tilde{e}_{\mathcal{N}_{i}}\right) f_{e_{\mathcal{N}}}\left(e_{\mathcal{N}_{i}}\right) \\
& +\left|\mathcal{N}_{i}\right| u_{i}^{T} R u_{i}+\frac{1}{2} \sum_{j \in \mathcal{N}_{i}} Q\left(e_{(i, j)}\right) \\
& -\frac{1}{2} \mathcal{W}_{\mathcal{N}_{i}}^{T} \nabla \sigma_{\mathcal{N}_{i}}\left(\tilde{e}_{\mathcal{N}_{i}}\right) f_{e_{\mathcal{N}}}\left(e_{\mathcal{N}_{i}}\right) \\
& -\mathcal{W}_{\mathcal{N}_{i}}^{T} \nabla \sigma_{\mathcal{N}_{i}}\left(\tilde{e}_{\mathcal{N}_{i}}\right) g_{\mathcal{N}_{i}}^{+}\left(e_{\mathcal{N}_{i}}\right) u_{i}^{\star} \\
& -\frac{1}{2} \nabla \varepsilon_{\mathcal{N}_{i}}\left(\tilde{e}_{\mathcal{N}_{i}}\right) f_{e_{\mathcal{N}}}\left(e_{\mathcal{N}_{i}}\right)-\nabla \varepsilon_{\mathcal{N}_{i}}\left(\tilde{e}_{\mathcal{N}_{i}}\right) g_{\mathcal{N}_{i}}^{-} u_{i}^{\star} \\
& -\left|\mathcal{N}_{i}\right| u_{i}^{\star T} R u_{i}^{\star}-\frac{1}{2} \sum_{j \in \mathcal{N}_{i}} Q\left(e_{(i, j)}\right) \\
& =\hat{\mathcal{W}}_{\mathcal{N}_{i}}^{c}{ }^{T} \nabla \sigma_{\mathcal{N}_{i}}\left(\tilde{e}_{\mathcal{N}_{i}}\right)\left(\frac{1}{2} f_{e_{\mathcal{N}}}\left(e_{\mathcal{N}_{i}}\right)+g_{\mathcal{N}_{i}}^{+}\left(e_{\mathcal{N}_{i}}\right) u_{i}\right) \\
& -\mathcal{W}_{\mathcal{N}_{i}}^{T} \nabla \sigma_{\mathcal{N}_{i}}\left(\tilde{e}_{\mathfrak{N}_{i}}\right)\left(\frac{1}{2} f_{e_{\mathcal{N}}}\left(e_{\mathcal{N}_{i}}\right)+g_{\mathcal{N}_{i}}^{+}\left(e_{\mathcal{N}_{i}}\right) u_{i}^{\star}\right. \\
& \left.-g_{\mathcal{N}_{i}}^{+}\left(e_{\mathcal{N}_{i}}\right) u_{i}+g_{\mathcal{N}_{i}}^{+}\left(e_{\mathcal{N}_{i}}\right) u_{i}\right)-\frac{1}{2} \nabla \varepsilon_{\mathcal{N}_{i}}\left(\tilde{e}_{\mathcal{N}_{i}}\right) f_{e_{\mathcal{N}}}\left(e_{\mathcal{N}_{i}}\right) \\
& -\nabla \varepsilon_{\mathcal{N}_{i}}\left(\tilde{e}_{\mathcal{N}_{i}}\right) g_{\mathcal{N}_{i}}^{-} u_{i}^{\star}+\left|\mathcal{N}_{i}\right| u_{i}^{T} R u_{i}-\left|\mathcal{N}_{i}\right| u_{i}^{\star T} R u_{i}^{\star} \\
& =-\tilde{\mathcal{W}}_{\mathcal{N}_{i}}^{c}{ }^{T} \nabla \sigma_{\mathcal{N}_{i}}\left(\tilde{e}_{\mathcal{N}_{i}}\right)\left(\frac{1}{2} f_{e_{\mathcal{N}}}\left(e_{\mathcal{N}_{i}}\right)+g_{\mathcal{N}_{i}}^{+}\left(e_{\mathcal{N}_{i}}\right) u_{i}\right) \\
& -\mathcal{W}_{\mathcal{N}_{i}}{ }^{T} \nabla \sigma_{\mathcal{N}_{i}}\left(\tilde{e}_{\mathcal{N}_{i}}\right) g_{\mathcal{N}_{i}}^{+}\left(e_{\mathcal{N}_{i}}\right) \tilde{u}_{i}-\frac{1}{2} \nabla \varepsilon_{\mathcal{N}_{i}}\left(\tilde{e}_{\mathcal{N}_{i}}\right) f_{e_{\mathcal{N}}}\left(e_{\mathcal{N}_{i}}\right) \\
& -\nabla \varepsilon_{\mathcal{N}_{i}}\left(\tilde{e}_{\mathcal{N}_{i}}\right) g_{\mathcal{N}_{i}}^{+} u_{i}^{\star}-\left|\mathcal{N}_{i}\right| \tilde{u}_{i}^{T} R\left(u_{i}^{\star}+u_{i}\right) \text {, }
\end{aligned}
$$

where

$$
\begin{aligned}
\tilde{\mathcal{W}}_{\mathcal{N}_{i}}^{c} & =\left[\cdots,\left(\beta_{(i, j)} \tilde{W}_{e}^{\left[\mathbf{x}_{h}\right]}+\tilde{\beta}_{(i, j)} \hat{W}_{e}^{\left[\mathbf{x}_{h}\right]}\right)^{T}, \cdots\right]^{T}: j \in \mathcal{N}_{i}, \\
& =\beta_{\mathcal{N}_{i}} \otimes I_{l} \tilde{W}_{\mathcal{N}_{i}}^{e}+\tilde{\beta}_{\mathcal{N}_{i}} \otimes I_{l} \hat{W}_{\mathcal{N}_{i}}^{e}, \quad \tilde{W}_{\mathcal{N}_{i}}^{e}, \hat{W}_{\mathcal{N}_{i}}^{e} \in \mathbb{R}^{l\left|\mathcal{N}_{i}\right|},
\end{aligned}
$$

where $I_{l} \in \mathbb{R}^{l \times l}, \quad \tilde{W}_{\mathcal{N}_{i}}^{e}=\left[\cdots, \tilde{W}_{e}^{\left[\mathbf{x}_{h}\right]^{T}}, \cdots\right]^{T}: j \in \mathcal{N}_{i}, \quad \hat{W}_{\mathcal{N}_{i}}^{e}=$ $\left[\cdots, \hat{W}_{e}^{\left[\mathbf{x}_{h}\right]^{T}}, \cdots\right]^{T}: j \in \mathcal{N}_{i}$, and we recast (57) to

$$
\begin{aligned}
\delta_{\mathcal{N}_{i}}= & -\hat{W}_{\mathcal{N}_{i}}^{e}{ }^{T} \tilde{\beta}_{\mathcal{N}_{i}}{ }^{T} \otimes I_{l} \nabla \sigma_{\mathcal{N}_{i}}\left(\tilde{e}_{\mathcal{N}_{i}}\right)\left(\frac{1}{2} f_{e_{\mathcal{N}}}\left(e_{\mathcal{N}_{i}}\right)+g_{\mathcal{N}_{i}}^{+}\left(e_{\mathcal{N}_{i}}\right) u_{i}\right) \\
& -\aleph_{\beta_{i}}-\aleph_{W_{i}}-\aleph_{\varepsilon_{i}}-\aleph_{R_{i}},
\end{aligned}
$$

where $\boldsymbol{\aleph}_{\varepsilon_{i}}, \aleph_{W_{i}}, \aleph_{R_{i}}$, and $\boldsymbol{\aleph}_{\beta_{i}}$ are bounded terms expressed as

$$
\begin{aligned}
\aleph_{\varepsilon_{i}} & =\frac{1}{2} \nabla \varepsilon_{\mathcal{N}_{i}}\left(\tilde{e}_{\mathcal{N}_{i}}\right) f_{e_{\mathcal{N}}}\left(e_{\mathcal{N}_{i}}\right)+\nabla \varepsilon_{\mathcal{N}_{i}}\left(\tilde{e}_{\mathcal{N}_{i}}\right) g_{\mathcal{N}_{i}}^{+} u_{i}^{\star} \\
\aleph_{W_{i}} & =\mathcal{W}_{\mathcal{N}_{i}}^{T} \nabla \sigma_{\mathcal{N}_{i}}\left(\tilde{e}_{\mathcal{N}_{i}}\right) g_{\mathcal{N}_{i}}^{+}\left(e_{\mathcal{N}_{i}}\right) \tilde{u}_{i} \\
\aleph_{R_{i}} & =\left|\mathcal{N}_{i}\right| \tilde{u}_{i}^{T} R\left(u_{i}^{\star}+u_{i}\right) \\
\aleph_{\beta_{i}} & =\tilde{W}_{\mathcal{N}_{i}}^{e} \beta_{\mathcal{N}_{i}}^{T} \otimes I_{l} \nabla \sigma_{\mathcal{N}_{i}}\left(\tilde{e}_{\mathcal{N}_{i}}\right)\left(\frac{1}{2} f_{e_{\mathcal{N}}}\left(e_{\mathcal{N}_{i}}\right)+g_{\mathcal{N}_{i}}^{+}\left(e_{\mathcal{N}_{i}}\right) u_{i}\right) .
\end{aligned}
$$

Let $\tilde{\beta}_{(i, j)}=\beta_{(i, j)}-\hat{\beta}_{(i, j)}$, we construct Lyapunov function $\mathcal{V}_{\beta}$ as follows

$$
\nu_{\beta}=\frac{1}{2 k_{\beta}} \sum_{i, j=1}^{N} \alpha_{i j}\left(\tilde{\beta}_{(i, j)}\right)^{2}
$$

and the time derivative of $\mathcal{V}_{\beta}$ is given by (62). Let

$$
\Re_{\mathcal{N}_{i}}=\hat{W}_{\mathcal{N}_{i}}^{e}{ }^{T} \tilde{\beta}_{\mathcal{N}_{i}}{ }^{T} \otimes I_{l} \nabla \sigma_{\mathcal{N}_{i}}\left(\tilde{e}_{\mathcal{N}_{i}}\right)\left(\frac{1}{2} f_{e_{\mathcal{N}}}\left(e_{\mathcal{N}_{i}}\right)+g_{\mathcal{N}_{i}}^{+}\left(e_{\mathcal{N}_{i}}\right) u_{i}\right),
$$

then, (62) can be recast to

$$
\dot{\mathcal{V}}_{\beta}=-\sum_{i=1}^{N}\left(\Re_{\mathcal{N}_{i}}\right)^{2}-\sum_{i=1}^{N} \Re_{\mathcal{N}_{i}}\left(\aleph_{\beta_{i}}+\aleph_{W_{i}}+\aleph_{\varepsilon_{i}}+\aleph_{R_{i}}\right),
$$

and given that $\hat{W}_{e}^{\left[\mathbf{x}_{h}\right]}$ is initialized through backward propagation in

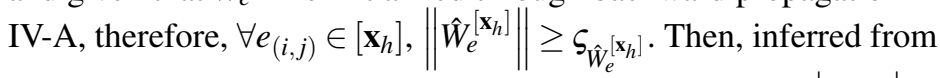
(64), there exists a bound $\varsigma_{\tilde{\beta}_{(i, j)}}$ such that $\dot{\mathcal{V}}_{\beta}<0$ when $\left|\tilde{\beta}_{(i, j)}\right|>$ $\varsigma_{\tilde{\beta}_{(i, j)}}: j \in \mathcal{N}_{i}$, and $\left|\tilde{\beta}_{(i, j)}\right|$ is thereby UUB. As $\left\|\tilde{W}_{e}^{\left[\mathbf{x}_{h}\right]}\right\|$ is UUB proved in Theorem 4, $\left\|\tilde{\mathcal{W}}_{(i, j)}^{\left[\mathbf{x}_{h}\right]}\right\|=\left\|\beta_{(i, j)} \tilde{W}_{e}^{\left[\mathbf{x}_{h}\right]}+\tilde{\beta}_{(i, j)} \hat{W}_{e}^{\left[\mathbf{x}_{h}\right]}\right\|$ is also UUB. 
Let $\tilde{S}_{(i, j)}^{a}=\hat{W}_{e}^{\left[\mathbf{x}_{h}\right]}-\hat{S}_{(i, j)}^{a}$, the time derivative of the Lyapunov function $\mathcal{V}_{(i, j)}^{a}=\frac{1}{2} \tilde{S}_{(i, j)}^{a}{ }^{T} \tilde{S}_{(i, j)}^{a}$ can be written as shown below:

$$
\begin{aligned}
\dot{\mathcal{V}}_{(i, j)}^{a}= & -\frac{k_{c} k_{a}\left|\delta_{e}^{\left[\mathbf{x}_{h}\right]}\right|\left\|\Gamma_{e}^{\left[\mathbf{x}_{h}\right]} \omega_{(i, j)}\right\|}{\left(\lambda_{e}^{\left[\mathbf{x}_{h}\right]}+\varepsilon_{a}\right)\left|1+\omega_{(i, j)}{ }^{T} \Gamma_{e}^{\left[\mathbf{x}_{h}\right]} \omega_{(i, j)}\right|} \tilde{S}_{(i, j)}^{a}{ }^{T} \tilde{S}_{(i, j)}^{a} \\
& -k_{c} \delta_{e}^{\left[\mathbf{x}_{h}\right]} \tilde{S}_{(i, j)}^{a}{ }^{T} \frac{\Gamma_{e}^{\left[\mathbf{x}_{h}\right]} \omega_{(i, j)}}{1+\omega_{(i, j)}{ }^{T} \Gamma_{e}^{\left[\mathbf{x}_{h}\right]} \omega_{(i, j)}} \\
\leq & -\frac{k_{c} k_{a}\left|\delta_{e}^{\left[\mathbf{x}_{h}\right]}\right|\left\|\Gamma_{e}^{\left[\mathbf{x}_{h}\right]} \omega_{(i, j)}\right\|}{\left(\lambda_{e}^{\left[\mathbf{x}_{h}\right]}+\varepsilon_{a}\right)\left|1+\omega_{(i, j)}{ }^{T} \Gamma_{e}^{\left[\mathbf{x}_{h}\right]} \omega_{(i, j)}\right|}\left\|\tilde{S}_{(i, j)}^{a}\right\|^{2} \\
& +\frac{k_{c}\left|\delta_{e}^{\left[\mathbf{x}_{h}\right]}\right|\left\|\Gamma_{e}^{\left[\mathbf{x}_{h}\right]} \omega_{(i, j)}\right\|}{\left|1+\omega_{(i, j)}{ }^{T} \Gamma_{e}^{\left[\mathbf{x}_{h}\right]} \omega_{(i, j)}\right|}\left\|\tilde{S}_{(i, j)}^{a}\right\|,
\end{aligned}
$$

and $\dot{\mathcal{V}}_{(i, j)}^{a}<0$ when $\left\|\tilde{S}_{(i, j)}^{a}\right\| \geq \frac{1+\varepsilon_{a}}{k_{a}}$. Thus, $\left\|\tilde{\mathcal{W}}_{(i, j)}^{a}\right\|=$ $\left\|\beta_{(i, j)}\left(\tilde{W}_{e}^{\left[\mathbf{x}_{h}\right]}+\tilde{S}_{(i, j)}^{a}\right)+\tilde{\beta}_{(i, j)} \hat{S}_{(i, j)}^{a}\right\|$ is UUB.

If the NN is ideal, then for $\forall \alpha_{i j}>0$ and $\left[\mathbf{x}_{h}\right] \subset \operatorname{Capt}_{\tilde{F}_{e}}(K, C) \backslash \mathbb{C}$, it is obvious that $\left\|\nabla \varepsilon_{(i, j)}^{\left[\mathbf{x}_{h}\right]}\left(\tilde{e}_{(i, j)}\right)\right\|=0$ and $\boldsymbol{\aleph}_{\varepsilon_{i}}=0$. The control error $\tilde{u}_{ \pm}$is given below:

$$
\begin{aligned}
\tilde{u}_{ \pm}= & \underset{u_{i j} \in\left[U_{2}\right]}{\arg \min }\left\{W_{e}^{\left[\mathbf{x}_{h}\right]^{T}} \nabla \sigma\left(\tilde{e}_{(i, j)}\right) g_{e}^{ \pm}\left(e_{(i, j)}\right) u_{i j}+u_{i j}^{T} R_{2} u_{i j}\right\} \\
& -\underset{u_{i j} \in\left[U_{2}\right]}{\arg \min }\left\{\hat{W}_{e}^{\left[\mathbf{x}_{h}\right]^{T}} \nabla \sigma\left(\tilde{e}_{(i, j)}\right) g_{e}^{ \pm}\left(e_{(i, j)}\right) u_{i j}+u_{i j}^{T} R_{2} u_{i j}\right\} .
\end{aligned}
$$

According to (44), we can deduce that

$$
\begin{aligned}
\dot{\mathcal{V}}_{e}^{\left[\mathbf{x}_{h}\right]}< & -k_{c} \frac{\left\|\omega_{(i, j)}\right\|^{2}\left\|\tilde{W}_{e}^{\left[\mathbf{x}_{h}\right]}\right\|^{2}}{1+\omega_{(i, j)} \Gamma_{e}^{\left[\mathbf{X}_{h}\right]} \omega_{(i, j)}}+2 k_{c} \frac{\left\|\omega_{(i, j)}\right\|\left\|\tilde{W}_{e}^{\left[\mathbf{x}_{h}\right]}\right\|}{1+\omega_{(i, j)}{ }^{T} \Gamma_{e}^{\left[\mathbf{x}_{h}\right]} \omega_{(i, j)}} \\
& \times\left(\left\|W_{e}^{\left[\mathbf{x}_{h}\right]^{T}} \nabla \sigma\left(\tilde{e}_{(i, j)}\right) g_{e}^{ \pm}\left(e_{(i, j)}\right)\right\|\left\|\tilde{u}_{ \pm}\right\|\right. \\
& \left.+\sigma_{\max }\left(R_{2}\right)\left\|u_{ \pm}^{\star}+u_{i j}\right\|\left\|\tilde{u}_{ \pm}\right\|\right) .
\end{aligned}
$$

Then, let

$$
\begin{aligned}
\varsigma_{\tilde{W}_{e}^{\left[\mathbf{x}_{h}\right]}}= & \frac{2\left\|W_{e}^{\left[\mathbf{x}_{h}\right]^{T}} \nabla \sigma\left(\tilde{e}_{(i, j)}\right) g_{e}^{ \pm}\left(e_{(i, j)}\right)\right\|\left\|\tilde{u}_{ \pm}\right\|}{\left\|\omega_{(i, j)}\right\|} \\
& +\frac{2 \sigma_{\max }\left(R_{2}\right)\left\|u_{ \pm}^{\star}+u_{i j}\right\|\left\|\tilde{u}_{ \pm}\right\|}{\left\|\omega_{(i, j)}\right\|},
\end{aligned}
$$

and when $\left\|\tilde{W}_{e}^{\left[\mathbf{x}_{h}\right]}\right\|>\varsigma_{\tilde{W}_{e}^{\left[\mathbf{x}_{h}\right]}}, \dot{V}_{e}^{\left[\mathbf{x}_{h}\right]}<0$. Thus, $\left\|\tilde{W}_{e}^{\left[\mathbf{x}_{h}\right]}\right\|$ is ultimately bounded by $\varsigma_{\tilde{W}_{e}\left[\mathbf{x}_{h}\right]}$ weighed by $\left\|\tilde{u}_{ \pm}\right\|$. By tuning $k_{c}$ and $k_{\lambda}$, $\boldsymbol{S}_{\tilde{u}_{ \pm}}^{\left[\mathbf{x}_{h}\right]}=\max _{e_{(i, j)} \in\left[\mathbf{x}_{h}\right]}\left\|\tilde{u}_{ \pm}\left(e_{(i, j)}\right)\right\|$ can be arbitrarily small after backward propagation, if the state resolution $\varepsilon_{X}$ in the propagation is small enough, as the trajectories of the estimated states $\hat{\phi}$ in box $\left[\mathbf{x}_{h}\right]$ discussed in (46) will be close to the optimal trajectories generated by backward propagation using PMP. Apart from that, $\hat{W}_{e}^{\left[\mathbf{x}_{h}\right]}$ is updated by on-line reinforcement learning without increasing $\varsigma_{\tilde{u}_{ \pm}}^{\left[\mathbf{x}_{h}\right]}$, as $\left\|\tilde{W}_{e}^{\left[\mathbf{x}_{l}\right]}\right\|$ asymptotically converges to its bound $\boldsymbol{\zeta}_{\tilde{W}_{e}\left[\mathbf{x}_{h}\right]}$. To summarize, a smaller set of resolutions $\varepsilon_{X}$ and larger parameters $k_{c}$ and $k_{\lambda}$ will lead to a smaller ultimate bound $\varsigma_{\tilde{W}_{e}^{\left[\mathbf{x}_{h}\right]}}$ of $\left\|\tilde{W}_{e}^{\left[\mathbf{x}_{h}\right]}\right\|$.

By adjusting the tuning parameter $k_{a}, \varsigma_{\tilde{S}_{a}}$ can be arbitrarily small when $t \rightarrow \infty$, such that $\hat{S}_{(i, j)}^{a}$ asymptotically converges to $\hat{W}_{e}^{\left[\mathbf{x}_{h}\right]}$ when $e_{(i, j)} \in\left[\mathbf{x}_{h}\right]$. Assuming that $\varepsilon_{X}$ is small enough, we have

$$
\begin{aligned}
& \forall\left[\mathbf{x}_{o}\right] \subset \operatorname{Capt}_{\tilde{F}_{e}}(K, C) \backslash \mathbb{C}, \quad\left[\mathbf{x}_{o}\right] \cap\left[\mathbf{x}_{h}\right] \neq \varnothing, \\
& \max _{o}\left\{\left\|\hat{W}_{e}^{\left[\mathbf{x}_{h}\right]}-\hat{W}_{e}^{\left[\mathbf{x}_{o}\right]}\right\|\right\} \rightarrow \varepsilon_{[\mathbf{x}]},
\end{aligned}
$$

where $\varepsilon_{[\mathbf{x}]}$ is a positive constant which can be arbitrarily small. If $k_{a}$ is sufficiently large, $\left\|\tilde{S}_{(i, j)}^{a}\right\|$ can be arbitrarily small for $\forall e_{(i, j)} \in$ $\operatorname{Capt}_{\tilde{F}_{e}}(K, C) \backslash \mathbb{C}$. We assume that $\varepsilon_{X}$ is sufficiently small, there exist $k_{c}, k_{\lambda}$ and $k_{a}$ such that the control error $\tilde{u}_{i}$ can be arbitrarily small. Then, based on the result of (64), the convergence of $\left\|\tilde{\beta}_{\mathcal{N}_{i}}\right\|$ reduces $\left\|\tilde{u}_{i}\right\|$, and meanwhile the bounded terms $\aleph_{\beta_{i}}, \boldsymbol{\aleph}_{W_{i}}, \boldsymbol{\aleph}_{\varepsilon_{i}}$, and $\aleph_{R_{i}}$ decrease due to the decay of $\left\|\tilde{u}_{i}\right\|$. When these bounded terms are eliminated, $\dot{\mathcal{V}}_{\beta}<0$ always holds, and $\left\|\tilde{\beta}_{\mathcal{N}_{i}}\right\| \rightarrow 0$. Given that

$$
\begin{aligned}
\left\|\tilde{\mathcal{W}}_{\mathcal{N}_{i}}^{c}\right\| & \leq\left\|\beta_{\mathcal{N}_{i}}\right\|\left\|\tilde{W}_{\mathcal{N}_{i}}^{e}\right\|+\left\|\tilde{\beta}_{\mathcal{N}_{i}}\right\|\left\|\hat{W}_{\mathcal{N}_{i}}^{e}\right\|, \\
\left\|\tilde{\mathcal{W}}_{\mathcal{N}_{i}}^{a}\right\| & \leq\left\|\beta_{\mathcal{N}_{i}}\right\|\left\|\tilde{S}_{\mathcal{N}_{i}}^{a}\right\|+\left\|\tilde{\beta}_{\mathcal{N}_{i}}\right\|\left\|\hat{S}_{\mathcal{N}_{i}}^{a}\right\|,
\end{aligned}
$$

where $\tilde{S}_{\mathcal{N}_{i}}^{a}=\left[\cdots, W_{e}^{\left[\mathbf{x}_{h}\right]}-\hat{S}_{(i, j)}^{a}, \cdots\right]^{T}: j \in \mathcal{N}_{i}$. Thus $\left\|\tilde{\mathcal{W}}_{\mathcal{N}_{i}}^{c}\right\|$ and $\left\|\tilde{\mathcal{W}}_{\mathcal{N}_{i}}^{a}\right\|$ can be arbitrarily small, and $u_{i}$ ultimately converge to $u_{i}^{\star}$.

\section{Simulation Results}

In this section, the simulations of autonomous vehicle networks are demonstrated. Consider the kinematic model of the $i$ th vehicle system in the global coordinate frame:

$$
\left\{\begin{array}{l}
\dot{x}_{i}=v_{i} \cos \left(\theta_{i}\right), \\
\dot{y}_{i}=v_{i} \sin \left(\theta_{i}\right), \\
\dot{\theta}_{i}=w_{i},
\end{array}\right.
$$

where $u_{i}=\left[v_{i}, w_{i}\right]^{T} \in[U]$ and $x_{i}, y_{i}, \theta_{i}, v_{i}, w_{i} \in \mathbb{R}$. Then, let $e_{(i, j)}=$ $\left[x_{(i, j)}, y_{(i, j)}, \theta_{(i, j)}\right]^{T}$, and the relative error system for two connected vehicles is expressed as

$$
\begin{aligned}
g_{e}^{\sharp}\left(e_{(i, j)}\right) & =\left[\begin{array}{cc}
-1 & y_{(i, j)} \\
0 & -x_{(i, j)} \\
0 & -1
\end{array}\right], \quad g_{e}^{b}\left(e_{(i, j)}\right)=\left[\begin{array}{cc}
\cos \left(\theta_{(i, j)}\right) & 0 \\
\sin \left(\theta_{(i, j)}\right) & 0 \\
1 & 0
\end{array}\right], \\
\dot{e}_{(i, j)} & =g_{e}^{+}\left(e_{(i, j)}\right) u_{i}+g_{e}^{-}\left(e_{(i, j)}\right) u_{j} .
\end{aligned}
$$

According to $(10), \psi_{C}\left(e_{(i, j)}\right), Q\left(e_{(i, j)}\right)$ and $R_{2}$ are selected as shown below:

$$
\begin{aligned}
\psi_{C}\left(e_{(i, j)}\right)= & 125 \operatorname{sign}\left(x_{(i, j)}\right)\left(x_{(i, j)}\right)^{3}+625\left(y_{(i, j)}\right)^{4} \\
& +6.32 \operatorname{sign}\left(\theta_{(i, j)}\right)\left(\theta_{(i, j)}\right)-1, \\
Q\left(e_{(i, j)}\right)= & e_{(i, j)} T\left[\begin{array}{ccc}
0.25 & 0 & 0 \\
0 & 0.5 & 0 \\
0 & 0 & 0.1621
\end{array}\right] e_{(i, j)}, \\
R= & {\left[\begin{array}{cc}
0.01 & 0 \\
0 & 0.01
\end{array}\right] }
\end{aligned}
$$




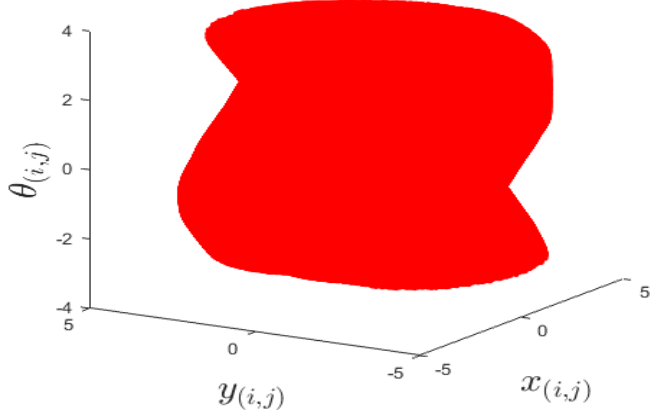

Fig. 2. Inner approximation of the capture basin $\operatorname{Capt}_{\tilde{F}_{e}}(K, C)$.

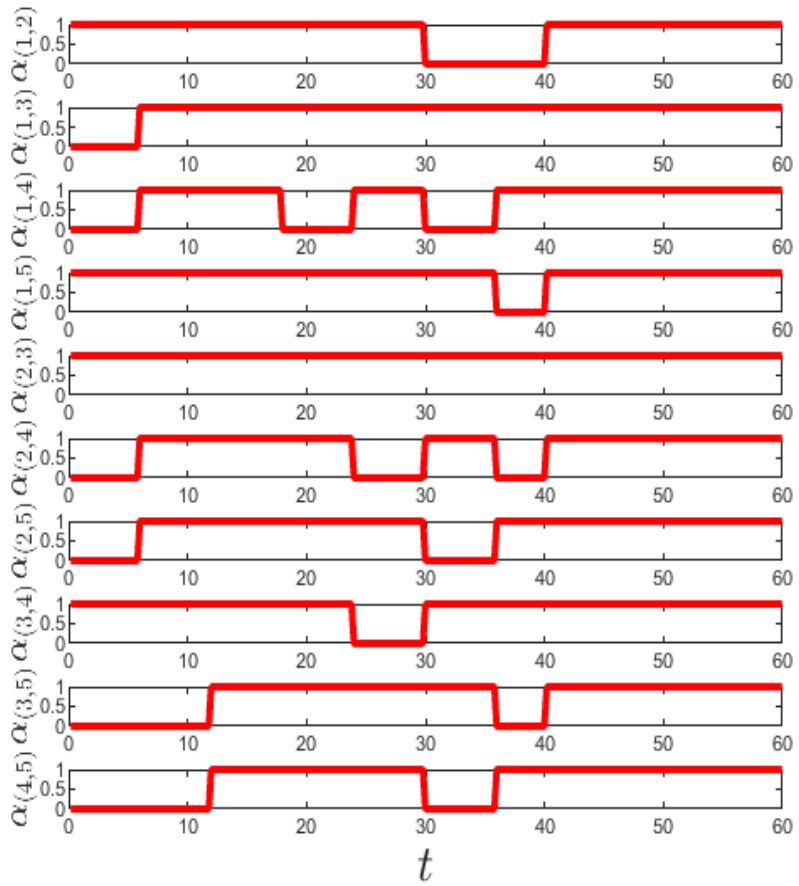

Fig. 3. Switching communication graph $\mathcal{A}(t)$.

and the control constraint $[U]$ and the communication zone $K$ are determined as

$$
\begin{aligned}
& \left.[U]=\left[\begin{array}{ll}
0.02 & 0.2
\end{array}\right] \quad\left[\begin{array}{ll}
-\frac{\pi}{10} & \frac{\pi}{10}
\end{array}\right]\right]^{T}, \\
& K=\left\{e_{(i, j)} \in X \mid \frac{1}{25}\left(x_{(i, j)}\right)^{2}+\frac{1}{25}\left(y_{(i, j)}\right)^{2}<1\right\} .
\end{aligned}
$$

and the resolution in backward propagation is set to $\varepsilon_{X}=$ $\left[0.05,0.05, \frac{\pi}{60}\right]^{T}$. The approximation result of the capture basin is depicted by its vertices shown in Fig. 2.

The switching graph is depicted in Fig. 3, in which $\alpha_{(i, j)}$ represents the undirected communication link between $v_{i}$ and $v_{j}$. Fig. 4 shows the 3-D plot of the states of five vehicles by the distributed control with the cooperative learning, and they converge at around $t=39 s$. The convergences of $\beta_{(i, j)}$ are shown in Fig. $5(\mathrm{a})$, and all the $\beta_{(i, j)}$ converge at $t=300 \mathrm{~s}$. The convergences of bellman errors $\delta_{\mathcal{N}_{i}}$ is shown in Fig. 5(b).

Given the case in Fig. 4, Fig. 6(a) illustrates the comparison of the global accumulative costs $J(T, e(0), u)$ and $\mathbf{J}(T, e(0), \mathbf{u})$, specified in (14), corresponding to the algorithms with and without the

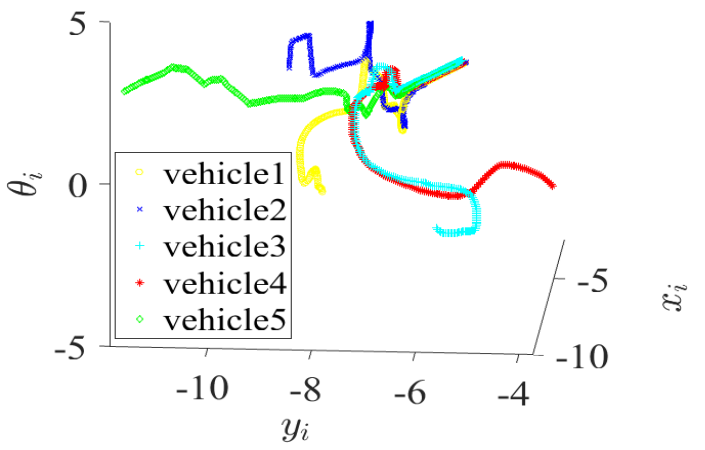

Fig. 4. The state convergences of five vehicles by the distributed control with the cooperative learning.

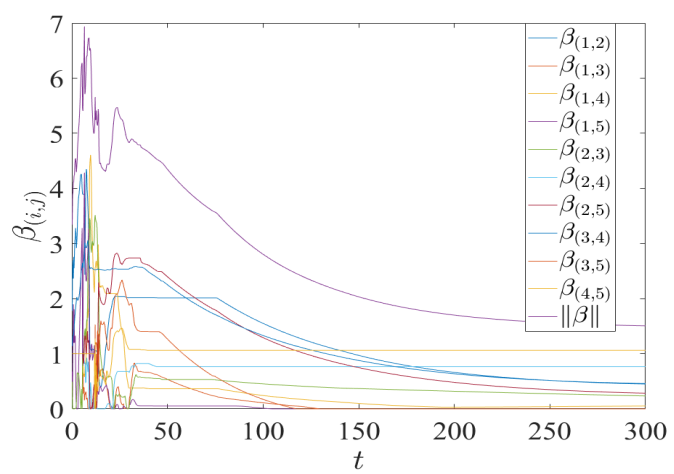

(a)

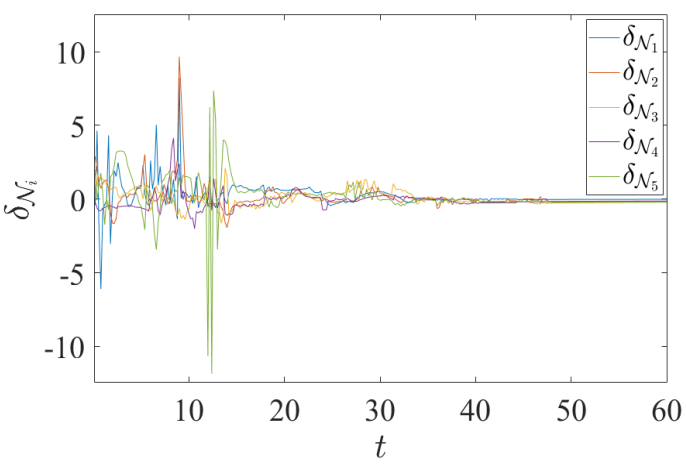

(b)

Fig. 5. Convergences of $\beta_{(i, j)}$ and bellman errors $\delta_{\mathfrak{N}_{i}}$ : (a) Convergences of $\beta_{(i, j)}$; (b) Convergences of bellman errors $\delta_{\mathcal{N}_{i}}$.

cooperative learning, respectively. Fig. 6(b) illustrates the global accumulative cost comparisons of four groups of simulations for different numbers of agents, and each group contains ten simulation cases. Defining $\mathbf{T}=\inf \left\{T \in(0, \infty) \mid \forall \alpha_{i j}=1, e_{(i, j)}(T) \in \mathbb{C}\right\}$ for a pair of cases to compare, the symbols represent $\frac{J(\mathbf{T}, e(0), u)}{\mathbf{J}(\mathbf{T}, e(0), \mathbf{u})}$.

\section{CONCLUSiOn AND FUture Work}

Based on the viability theory, we have proposed a novel distributed control protocol for nonlinear systems with bounded control by formulating distributed control problems to differential graphical games. Our algorithm involves the game theory, optimal theory, viability theory, interval methods, reinforcement learning technique, etc. We adopted the reinforcement learning technique 


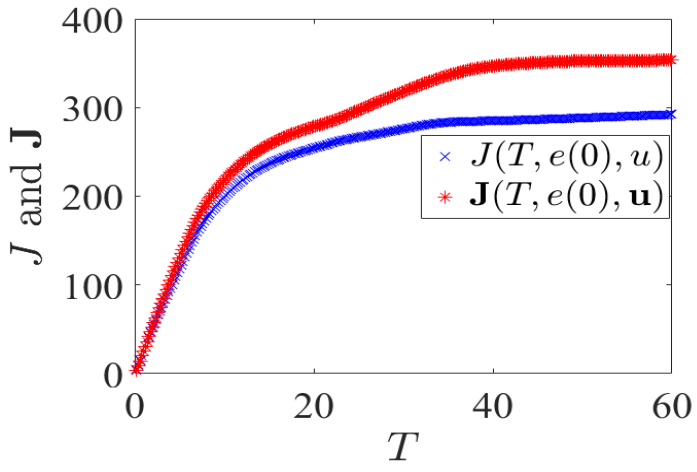

(a)

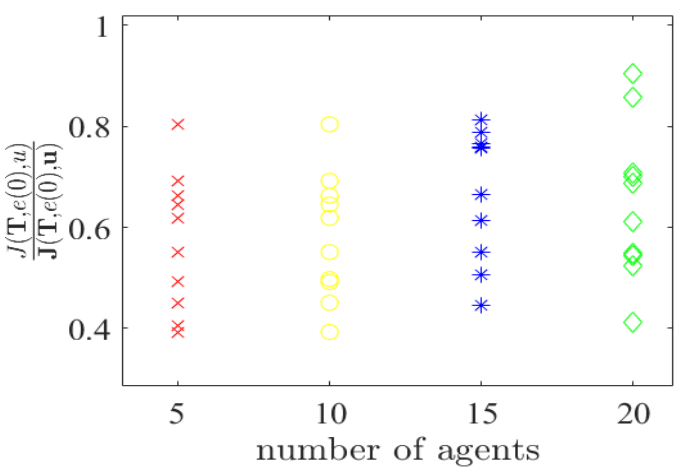

(b)

Fig. 6. Global cost comparison between the algorithms with and without the cooperative learning: (a) Cost comparison of a pair of cases; (b) Cost comparisons of four groups of simulations for different numbers of agents.

when approximating the capture basin. The off-line computation of the capture basin generates a priori knowledge and characterizes the feasible learning scope for the on-line reinforcement learner. By using on-line cooperative reinforcement learning, the dimensionality issue of implementing the tools of the viability theory has been overcome. We approximated the Pareto optimality of the graphical game rather than the Nash equilibrium, thus achieving an optimal convergence cost of states in a global sense. Our future work will focus on differential graphical games among groups of vehicles with uncertainties, deep distributed reinforcement learning, and the viability property of complex network systems.

\section{REFERENCES}

[1] C. Yuan, S. Licht, and H. He, "Formation learning control of multiple autonomous underwater vehicles with heterogeneous nonlinear uncertain dynamics," IEEE Transactions on Cybernetics, vol. 48, no. 10, pp. 2920 2934, 2018

[2] B. Liu, Z. Ding, and C. Lv, "Distributed training for multi-layer neural networks by consensus," IEEE transactions on neural networks and learning systems, 2019.

[3] F. L. Lewis, H. Zhang, K. Hengster Movric, and A. Das, Cooperative control of multi-agent systems: optimal and adaptive design approaches. Springer Science \& Business Media, 2013.

[4] G. Guo and D. Li, "Adaptive sliding mode control of vehicular platoons with prescribed tracking performance," IEEE Transactions on Vehicular Technology, vol. 68, no. 8, pp. 7511-7520, 2019.

[5] G. Guo and L. Wang, "Control over medium-constrained vehicular networks with fading channels and random access protocol: a networked systems approach," IEEE Transactions on Vehicular Technology, vol. 64, no. 8, pp. 3347-3358, 2014.

[6] S. Wen and G. Guo, "Sampled-data control for connected vehicles with markovian switching topologies and communication delay," IEEE Transactions on Intelligent Transportation Systems, 2019.
[7] J. Hu, P. Bhowmick, and A. Lanzon, "Distributed adaptive time-varying group formation tracking for multi-agent systems with multiple leaders on directed graphs," IEEE Transactions on Control of Network Systems, 2019.

[8] D. Vickrey and D. Koller, "Multi-agent algorithms for solving graphical games," in AAAI/IAAI, 2002, pp. 345-351.

[9] J. Von Neumann and O. Morgenstern, "Theory of games and economic behavior, 2nd rev," 1947.

[10] D. Fudenberg and J. Tirole, "Game theory cambridge ma," 1991.

[11] M. K. Michael and M. L. Littman, "Graphical models for game theory," in In UAI. Citeseer, 2001.

[12] M. Ye, "Distributed robust seeking of nash equilibrium for networked games: An extended state observer-based approach," IEEE Transactions on Cybernetics, pp. 1-12, 2020.

[13] Y. Lv and X. Ren, "Approximate nash solutions for multiplayer mixed-zerosum game with reinforcement learning," IEEE Transactions on Systems, Man, and Cybernetics: Systems, vol. 49, no. 12, pp. 2739-2750, 2019.

[14] K. Lu, G. Jing, and L. Wang, "Distributed algorithms for searching generalized nash equilibrium of noncooperative games," IEEE Transactions on Cybernetics, vol. 49, no. 6, pp. 2362-2371, 2019.

[15] M. Ye and G. Hu, "Distributed nash equilibrium seeking in multiagent games under switching communication topologies," IEEE Transactions on Cybernetics, vol. 48, no. 11, pp. 3208-3217, 2018.

[16] P. Cardaliaguet, M. Quincampoix, and P. Saint Pierre, "Differential games through viability theory: Old and recent results," in Advances in Dynamic Game Theory. Springer, 2007, pp. 3-35.

[17] M. Bardi, T. Raghavan, and T. Parthasarathy, Stochastic and differential games: theory and numerical methods. Springer Science \& Business Media, 2012, vol. 4.

[18] J. P. Aubin, "Victory and defeat in differential games," in Modeling and Control of Systems. Springer, 1989, pp. 337-347.

[19] — "Differential games: a viability approach," SIAM Journal on Control and Optimization, vol. 28, no. 6, pp. 1294-1320, 1990.

[20] P. Cardaliaguet, "A differential game with two players and one target," SIAM Journal on Control and Optimization, vol. 34, no. 4, pp. 1441-1460, 1996.

[21] P. Cardaliaguet and S. Plaskacz, "Viability and invariance for differential games with applications to hamilton-jacobi-isaacs equations," Banach Center Publications, vol. 35, no. 1, pp. 149-158, 1996.

[22] P. Cardaliaguet, M. Quincampoix, and P. Saint-Pierre, "Optimal times for constrained nonlinear control problems without local controllability," Applied Mathematics and Optimization, vol. 36, no. 1, p. 21, 1997.

[23] J. P. Aubin and N. Seube, "Conditional viability for impulse differential games," Annals of Operations Research, vol. 137, no. 1, pp. 269-297, 2005.

[24] V. Patsko and M. Quincampoix, "Preface: Dgaa special issue on pursuitevasion games and differential games with incomplete information," Dynamic Games and Applications, vol. 9, no. 3, pp. 569-572, 2019.

[25] Y. Averboukh, "A stability property in mean field type differential games," arXiv preprint arXiv:1903.11152, 2019.

[26] A. Hochart, "Unique ergodicity of deterministic zero-sum differential games," arXiv preprint arXiv:1908.03643, 2019.

[27] V. Ukhobotov and I. Izmestev, "Impulse differential game with a mixed constraint on the choice of the control of the first player," Proceedings of the Steklov Institute of Mathematics, vol. 304, no. 1, pp. S161-S174, 2019.

[28] B. Peng, A. Stancu, and S. Dang, "Computation of discriminating kernel and robust capture basin with regulation map by interval methods," International Journal of Robust and Nonlinear Control, vol. 29, no. 11, pp. 3737-3755, 2019.

[29] L. Jaulin, Applied interval analysis: with examples in parameter and state estimation, robust control and robotics. Springer Science \& Business Media, 2001, vol. 1.

[30] D. Monnet, J. Ninin, and L. Jaulin, "Computing an inner and an outer approximation of the viability kernel," Reliable Computing, vol. 22, 2016.

[31] M. Lhommeau, L. Jaulin, and L. Hardouin, "Capture basin approximation using interval analysis," International Journal of Adaptive Control and Signal Processing, vol. 25, no. 3, pp. 264-272, 2011.

[32] J. P. Aubin, A. M. Bayen, and P. Saint Pierre, Viability theory: new directions. Springer Science \& Business Media, 2011.

[33] J. P. Aubin and F. Catté, "Bilateral fixed-points and algebraic properties of viability kernels and capture basins of sets," Set-Valued Analysis, vol. 10, no. 4, pp. 379-416, 2002 .

[34] R. Kamalapurkar, P. Walters, J. Rosenfeld, and W. Dixon, "Differential graphical games," in Reinforcement Learning for Optimal Feedback Control. Springer, 2018, pp. 149-193.

[35] H. L. Stalford, "Criteria for pareto-optimality in cooperative differential games," Journal of Optimization Theory and Applications, vol. 9, no. 6, pp 391-398, Jun 1972. [Online]. Available: https://doi.org/10.1007/BF00934739

[36] R. Olfati Saber and R. M. Murray, "Distributed cooperative control of multiple vehicle formations using structural potential functions," in IFAC World Congress, vol. 15, no. 1. Citeseer, 2002, pp. 242-248.

[37] P. A. Ioannou and J. Sun, Robust adaptive control. Courier Corporation, 2012. 